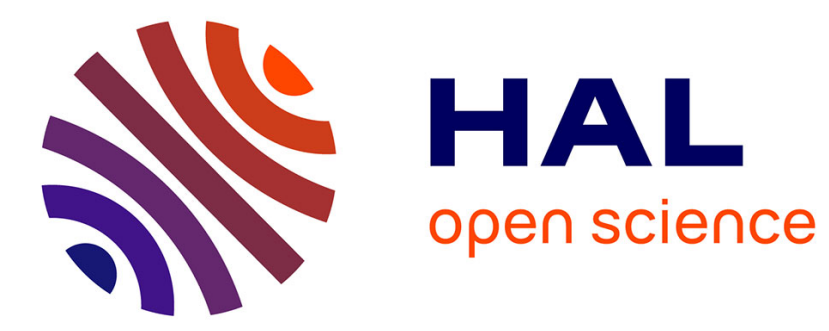

\title{
Space Variant Blind Image Restoration
}

Saima Ben Hadj, Laure Blanc-Féraud, Gilles Aubert

\section{To cite this version:}

Saima Ben Hadj, Laure Blanc-Féraud, Gilles Aubert. Space Variant Blind Image Restoration . SIAM Journal on Imaging Sciences, 2014, 7, pp.2196 - 2225. 10.1137/130945776 . hal-01096491

\section{HAL Id: hal-01096491 \\ https://hal.inria.fr/hal-01096491}

Submitted on 17 Dec 2014

HAL is a multi-disciplinary open access archive for the deposit and dissemination of scientific research documents, whether they are published or not. The documents may come from teaching and research institutions in France or abroad, or from public or private research centers.
L'archive ouverte pluridisciplinaire HAL, est destinée au dépôt et à la diffusion de documents scientifiques de niveau recherche, publiés ou non, émanant des établissements d'enseignement et de recherche français ou étrangers, des laboratoires publics ou privés. 


\title{
Space Variant Blind Image Restoration*
}

\author{
Saima Ben Hadj ${ }^{\dagger}$, Laure Blanc-Féraud ${ }^{\dagger}$, and Gilles Aubert ${ }^{\ddagger}$
}

\begin{abstract}
We are interested in blind restoration of optical space variant blurred Poissonian images. For example, blur variation is due to refractive index mismatch in three-dimensional fluorescence microscopy or due to atmospheric turbulence in astrophysical images. In this work, the space variant point spread function (PSF) is approximated by a convex combination of a set of space invariant blurring functions. The latter is jointly estimated with the image by optimizing a given criterion including $l_{1}$ and $l_{2}$ norms for regularizing the image and the PSFs. We prove, in the continuous setting, the existence of a solution to this optimization problem. We then propose an alternating optimization algorithm based on a scaled gradient projection method. We show the efficiency of the proposed method on simulated and real optical images.
\end{abstract}

Key words. blind restoration, space variant blur, PSF, SGP algorithm

AMS subject classifications. 94A08, 68U10, 34A12, 35A01, 74G25, 65B99, 65K10, 49K35, 46N60

DOI. $10.1137 / 130945776$

\section{Introduction.}

1.1. General framework and state of the art. We are interested in blind restoration of optical space variant blurred Poissonian images. The blur is inherent to optical instruments basically due to light diffraction and defocusing limits. This blur may be varying in the image for different reasons, such as atmospheric turbulence in astrophysical imaging, moving objects in a real scene, or refractive index variation in three-dimensional (3D) fluorescence microscopy. In case of low count levels, optical images are intrinsically noisy. From a mathematical point of view, the observed image $g$ that we are interested in is modeled by

$$
g \sim \mathcal{P}(H(f)),
$$

where $f$ is the image to be reconstructed, $H$ is the space variant blur operator, and $\mathcal{P}$ is the Poisson statistic. Knowing only the observation $g$, our goal is to recover the sharp image $f$. In this case, in real imaging, the blur depends on uncontrollable imaging conditions and thus is unknown. Many blind restoration methods have been proposed in the literature for that purpose. Most of them are carried under the space-invariance assumption of the blur, which reduces the problem complexity since the blur operator is a convolution. In fact, computations are significantly reduced by using the Fourier transform.

\footnotetext{
* Received by the editors November 11, 2013; accepted for publication (in revised form) July 28, 2014; published electronically November 11, 2014.

http://www.siam.org/journals/siims/7-4/94577.html

${ }^{\dagger}$ Morphème Research Group I3S/CNRS/INRIA/UNSA/IBDC, 06903 Sophia Antipolis Cedex, France (saima. benhadj@gmail.com, laure.blanc_feraud@inria.fr). The first author's work was supported by the ANR DIAMOND project.

${ }^{\ddagger}$ Laboratoire J.A. Dieudonne, UMR 6621 CNRS, University of Nice Sophia Antipolis, 06108 Nice Cedex 2, France (gilles.aubert@unice.fr).
} 
Nevertheless, dealing with a space variant blur (the convolution is no longer a valid representation), the system response or the point spread function (PSF) is different at each point. This makes computations prohibitively extensive. Few blind restoration methods have been proposed for this problem. Most of them are extensions of blind deconvolution methods. Let us recall some of these methods.

The first proposed methods were considering a piecewise invariant PSF. The image is thus segmented into subimages where the blur is assumed to be invariant; see $[22,28]$. A blind deconvolution method is then applied on each of these subimages separately. However, these methods lead to decomposition artifacts on the reconstructed image.

More recent methods consider a smoothly varying blur where the space variant PSF is modeled by a combination of space invariant ones. Two different approximation models of the space variant PSF have been developed in the literature, one in [32, 24, 25] and the other in $[4,29]$. These models differ in the way of interpolating the space invariant PSFs. Study and comparison of these models are discussed in $[7,15]$. Nevertheless, none of these aforementioned methods automatically estimates the space invariant PSF locations or the different functions used to compute the space variant PSFs from the space invariant PSFs. They usually assume that combination functions and space invariant PSF locations are known. In [3], an activecontour based method is proposed to detect the region boundaries where the blur could be considered space invariant. However, in this method, the blur variation between neighboring regions is supposed to be significant. In this work, both a blind restoration and smooth blur variations are considered. The space invariant PSF locations and combination functions are thus empirically fixed.

In some other works, such as [9], the PSF is estimated at every point of the image. This actually leads to an accurate result but with a huge computational time. The application of these methods to big images such as in 3D microscopy is thus prohibitive.

In essence, existing blind restoration methods differ in the way of regularizing the PSF and the image in order to compensate for the severe lack of information. For example, in [45], the authors consider a smoothness regularization for both the image and the PSF. In [33], the authors reduce the degree of freedom of the problem by using a parameterized PSF model. In [3], the authors use the Mumford-Shah model for the image regularization. Recent contributions on blind restoration can be found in [43, 48, 35].

1.2. Main contribution. In this paper, we propose a solution for the blind restoration of optical space variant blurred Poissonian images. In order to avoid the extensive computations due to the space variant blur, we approximate the space variant PSF by a convex combination of a set of space invariant PSFs $h^{1}, \ldots, h^{M}$, with $M \geq 1$ the number of PSFs. Thanks to this approximation, the blind restoration problem boils down to the estimation of the image $f$ and the set of space invariant PSFs $h^{1}, \ldots, h^{M}$. For that, we propose within a joint maximum a posteriori (JMAP) framework a criterion to be optimized w.r.t. $\left(f, h^{1}, \ldots, h^{M}\right)$ of the form

$$
J\left(f, h^{1}, \ldots, h^{M}\right)=J^{d}\left(f, h^{1}, \ldots, h^{M}\right)+J_{f}^{p}(f)+J_{h}^{p}\left(h^{1}, \ldots, h^{M}\right),
$$

$J^{d}\left(f, h^{1}, \ldots, h^{M}\right)$ being a data fidelity term arising from the considered image formation model. $J_{f}^{p}(f)$ and $J_{h}^{p}\left(h^{1}, \ldots, h^{M}\right)$ are, respectively, prior terms on the image and the PSF set. We use $l_{1}$ and $l_{2}$ norms, respectively, to define $J_{f}^{p}(f)$ and $J_{h}^{p}\left(h^{1}, \ldots, h^{M}\right)$ (cf. subsection 
2.4). We prove in Appendix A the existence of a solution of the optimization problem.

Then, we propose an algorithm for the numerical optimization of the considered criterion. We adopt an alternating optimization scheme in which we use the fast scaled gradient projection (SGP) algorithm [11, 47] for the elementary optimizations. We show in various numerical tests the interest of our method on different applications, namely digital camera and $3 \mathrm{D}$ confocal microscopy.

The paper is organized as follows: Section 2 is devoted to notation, definitions, and problem formulation. In section 3, we describe the proposed optimization algorithm based on the SGP algorithm. Experimental tests are presented in section 4. The paper concludes in section 5 with a summary and some perspectives of this work.

2. Problem formulation. The goal of this section is to define an appropriate criterion to be minimized for solving the blind restoration problem. For that, we first present the image formation model. We then introduce some preliminary notation and an important lemma regarding the properties of the PSF normalization and the flux conservation. Then, we present the approximation model for the space variant blur considered in this work. We finally derive the JMAP criterion to be minimized.

2.1. Image formation model. Before presenting the image formation model that we consider in this paper, we need to introduce the following notation.

\subsubsection{Notation.}

- $\mathcal{O}$ is a bounded set of $\mathbb{R}^{n}$ (with $n=2,3$ the image dimension), standing for the object space (i.e., the true scene).

- $\mathcal{I}$ is a bounded set of $\mathbb{R}^{n}$ standing for the image space (i.e., the observed scene).

- $f \in \mathcal{L}^{1}\left(\mathcal{O}, \mathbb{R}^{+}\right)$stands for the unknown intensity image, where $\mathcal{L}^{1}$ is the set of integrable functions from $\mathcal{O}$ to $\mathbb{R}^{+}$.

- $H: \mathcal{L}^{1}\left(\mathcal{O}, \mathbb{R}^{+}\right) \rightarrow \mathcal{L}^{1}\left(\mathcal{I}, \mathbb{R}^{+}\right)$is the blur operator.

- $k \in \mathcal{L}^{1}\left(\mathcal{I}, \mathbb{R}^{+}\right)$is the blurred version of $f$, i.e., $k=H(f)$.

- $\mathcal{I}_{d}$ is a bounded set of $\mathbb{N}^{n}, n=2,3$, corresponding to the support of the discrete image recorded at the sensor.

- $k_{d}: \mathcal{I}_{d} \rightarrow \mathbb{R}^{+}$is a discrete version of the blurred image $k$. For each pixel/voxel $j \in \mathcal{I}_{d}(j)$ is the recorded rate of photons in an elementary region $R_{j} \subset \mathbb{R}^{n}$, i.e., $k_{d}(j)=\int_{R_{j}} k(u) \mathrm{d} u=\int_{R_{j}} H(f)(u) \mathrm{d} u \forall j \in \mathcal{I}_{d}$.

- $g: \mathcal{I}_{d} \rightarrow \mathbb{N}$ is the discrete measurement vector of photon counts, i.e., $g \sim \mathcal{S}\left(k_{d}\right)$, where $\mathcal{S}$ is the measurement statistic.

We give in the following two subsections the expression of the blur operator $H$ and the measurement statistic $\mathcal{S}$.

2.1.2. Blur model. Denote by $h_{H} \in L^{1}(\mathcal{I} \times \mathcal{O})$ the space variant PSF; i.e., $h_{H}\left(., u^{\prime}\right)$ refers to the system response to a point source placed at $u^{\prime}$, a location in the object space $\mathcal{O}$. The space variant blurred image can be modeled by

$$
k(u)=H(f)(u)=\int_{\mathcal{O}} h_{H}\left(u, u^{\prime}\right) f\left(u^{\prime}\right) d u^{\prime} \quad \forall u \in \mathcal{I} .
$$

Due to its huge computational cost, the above model cannot be used in an iterative estimation process. We present in subsection 2.3 an approximation of that model. 
2.1.3. Measurement statistic. First, a background noise is usually present in astrophysical and microscopy images [40]. For example, in fluorescence microscopy, the background noise is due to autofluorescence of the medium or to the reflecting and scattering fluorescence of the specimen. Similarly, in astronomy, the background noise comes from the diffusion of the incoming light from nearby sources from the sky or from the telescope itself. In fact, even if no visible astronomical objects are present in a given part of the sky, there is always some low luminosity present, due to light diffusion from the atmosphere. This kind of noise can be assimilated to a nonnegative constant $b_{g}>0$ that can be estimated from a dark area of the observed image which does not contain a signal [30]. Thus, $b_{g}$ is assumed to be known in the model.

Second, astronomical and confocal microscopy images have an inherent Poissonian statistic due to the stochastic nature of photon counts at sensors. The observed image at each pixel/voxel $j \in \mathcal{I}_{d}$ is a realization of a Poisson statistic $\mathcal{P}$ of mean and variance equal to $k_{d}(j)+b_{g}:$

$$
g(j) \sim \mathcal{P}\left(k_{d}(j)+b_{g}\right) .
$$

That is, the conditional probability of $g(j)$ is written as follows:

$$
\operatorname{Pr}\left(g(j) \mid k_{d}(j)+b_{g}\right)=\frac{\left(k_{d}(j)+b_{g}\right)^{g(j)} e^{-\left(k_{d}(j)+b_{g}\right)}}{g(j) !} .
$$

The observations conditionally to the model $\left(g(j) \mid k_{d}(j)+b_{g}\right)$ being independent and identically distributed, the joint conditional probability of the whole image can be written as follows:

$$
\begin{aligned}
\operatorname{Pr}(g \mid f, H) & =\prod_{j \in \mathcal{I}_{d}} \operatorname{Pr}\left(g(j) \mid k_{d}(j)+b_{g}\right) \\
& =\prod_{j \in \mathcal{I}_{d}} \frac{\left(k_{d}(j)+b_{g}\right)^{g(j)} e^{-\left(k_{d}(j)+b_{g}\right)}}{g(j) !} .
\end{aligned}
$$

2.2. Notation and a preliminary lemma. In this work, we look for reconstructing an image of the same support as the observation $g$. Without loss of generality, we consider $\mathcal{O}=\mathcal{I}=[-1,1]^{n}, n=2,3$, the support of the observed two-dimensional (2D) or 3D image, and $B(O, R)$ a closed disc or sphere centered at the origin $O$, of radius $R \in[0,1]$. Consider the following spaces:

- $H^{1}(\mathcal{I})=\left\{h \in L^{2}(\mathcal{I}), \nabla h \in L^{2}(\mathcal{I})^{n}\right\}$.

- $\mathcal{H}=\left\{h \in H^{1}(\mathcal{I}) ; h \geq 0 ; \int_{\mathcal{I}} h(u) d u=1 ; \operatorname{supp}(h) \subset B(O, R)\right\}$, where $\operatorname{supp}(h)$ refers to the support of $h$. When $\int_{\mathcal{I}} h(u) d u=1$, the PSF $h$ is said to be normalized on $\mathcal{I}$.

- $\mathcal{F}=\left\{f \in B V\left(\mathbb{R}^{n}\right) ; f \geq 0 ; \mathcal{I}\right.$-periodic $\}$, where $B V\left(\mathbb{R}^{n}\right)$ is the space of functions of bounded variations; see [2].

- $\mathcal{F}_{c}=\left\{f \in \mathcal{F} ; \int_{\mathcal{I}} f(u) d u=c\right\}$, where $0<c<+\infty$ is a positive constant.

In the case of space invariant blur, the blurred image on a bounded set $\mathcal{I}$ is assumed to be obtained by convolving the image $f$ with a space invariant PSF that we denote by $h$. From a practical point of view, the convolution is computed in the Fourier domain. Consequently, the 
image $f$ is assumed to be infinite and periodic of period $\mathcal{I}$. That is why we consider $\mathcal{I}$-periodic functions in $\mathcal{F}$. Then, the convolution operation is defined for $f \in \mathcal{F}$ and $h \in H^{1}(\mathcal{I})$ :

$$
h * f(u)=\int_{\mathbb{R}^{n}} h\left(u-u^{\prime}\right) f\left(u^{\prime}\right) d u^{\prime}=\int_{\mathbb{R}^{n}} f\left(u-u^{\prime}\right) h\left(u^{\prime}\right) d u^{\prime} \quad \forall u \in \mathcal{I} .
$$

We consider in this work a photon conservative optical system. That is, the number of photons entering the optical system is the same as the number of photons leaving the optical system. This property is known as flux conservation. We state in Lemma 2.1 that this property is equivalent to the PSF normalization in the periodic case (the proof is elementary and hence omitted). In fact, flux conservation and PSF normalization are important for reducing the ambiguity due to the ill-posedness of the problem. For example, by imposing a PSF normalization, one can discard many possible PSFs obtained by rescaling the true one.

Lemma 2.1. Let $f \in \mathcal{F}$, let $h \in H^{1}(\mathcal{I})$, and let $\operatorname{supp}(h) \subset B(O, R)$; then we have

$$
\int_{\mathcal{I}} f * h(u) d u=\int_{\mathcal{I}} f(u) d u \Longleftrightarrow \int_{B(O, R)} h(u) d u=1 .
$$

2.3. Approximation model for the space variant PSF. Approximate space variant blur models based on a linear interpolation of a set of space invariant PSFs were previously studied and compared in $[7,15]$. In this work, we consider the approximation model similar to that proposed in $[24,25,32]$ :

$$
k(u)=\sum_{1 \leq i \leq M} h^{i} *\left(\psi^{i} \cdot f\right)(u) \quad \forall u \in \mathcal{I}
$$

where "." is a pointwise multiplication, $f \in \mathcal{F},\left\{h^{i} \in \mathcal{H}, 1 \leq i \leq M\right\}$ is a set of space invariant PSFs, and $\left\{\psi^{i}: \mathbb{R}^{n} \rightarrow[0,1], 1 \leq i \leq M\right\}$ is a set of weighting functions verifying $\sum_{1 \leq i \leq M} \psi^{i}\left(u^{\prime}\right)=1 \forall u^{\prime} \in \mathbb{R}^{n}$. Each weighting function is associated with a space invariant PSF allowing smooth transitions between neighboring space invariant PSFs. According to the variations of the considered weighting functions $\psi^{i}, i=1, \ldots, M$, the transitions between the different space invariant PSFs is more or less smooth. Examples of functions $\psi^{i}$ are given in section 4. The space variant PSF associated with model (2.7) is expressed as

$$
h_{H}\left(u, u^{\prime}\right)=\sum_{1 \leq i \leq M} \psi^{i}\left(u^{\prime}\right) h^{i}\left(u-u^{\prime}\right) \quad \forall u \in \mathcal{I}, u^{\prime} \in \mathbb{R}^{n} .
$$

A different approximation model was used in [4, 29], where the weighting functions $\psi^{i}$ vary with the image coordinates $u$. It was shown in $[7,15]$ that such a model is less realistic than model (2.7). We can easily verify that the space variant PSF given by (2.8) is normalized and the flux conservation property is satisfied when the space invariant PSFs $h^{i}, i=1, \ldots, M$, are normalized (see Lemmas 2.2 and 2.3). This is not the case, however, for the other model considered in $[4,29]$.

Lemma 2.2. Let $f \in L^{2}(\mathcal{I})$, let $h^{i} \in \mathcal{H}$, let $1 \leq i \leq M$, and let $B\left(u^{\prime}, R\right)$ be a bounded disc or sphere of radius $R \in[0,1]$, centered on $u^{\prime} \in \mathbb{R}^{n}$; then we have the result

$$
\int_{B\left(u^{\prime}, R\right)} \sum_{1 \leq i \leq M} \psi^{i}\left(u^{\prime}\right) h^{i}\left(u-u^{\prime}\right) d u=1 \quad \forall u^{\prime} \in \mathbb{R}^{n} .
$$


Proof.

$$
\begin{aligned}
\int_{B\left(u^{\prime}, R\right)} \sum_{1 \leq i \leq M} \psi^{i}\left(u^{\prime}\right) h^{i}\left(u-u^{\prime}\right) d u & =\sum_{1 \leq i \leq M} \psi^{i}\left(u^{\prime}\right) \int_{B\left(u^{\prime}, R\right)} h^{i}\left(u-u^{\prime}\right) d u \\
& =\sum_{1 \leq i \leq M} \psi^{i}\left(u^{\prime}\right) \int_{B(O, R)} h^{i}(t) d t \\
& =\sum_{1 \leq i \leq M} \psi^{i}\left(u^{\prime}\right) \\
& =1 .
\end{aligned}
$$

Lemma 2.3. Let $f \in L^{2}(\mathcal{I})$, let $h^{i} \in \mathcal{H}$, and let $1 \leq i \leq M$; then we have

$$
\int_{\mathcal{I}} \sum_{1 \leq i \leq M} h^{i} *\left(\psi^{i} \cdot f\right)(u) d u=\int_{\mathcal{I}} f(u) d u .
$$

Proof. We have

$$
\int_{\mathcal{I}} \sum_{1 \leq i \leq M} h^{i} *\left(\psi^{i} \cdot f\right)(u) d u=\sum_{1 \leq i \leq M} \int_{\mathcal{I}} h^{i} *\left(\psi^{i} \cdot f\right)(u) d u
$$

Moreover, as $h^{i}, 1 \leq i \leq M$, are normalized, we obtain from Lemma 2.1

$$
\begin{aligned}
\sum_{1 \leq i \leq M} \int_{\mathcal{I}} \psi^{i}(u) \cdot f(u) d u & =\int_{\mathcal{I}} \sum_{1 \leq i \leq M} \psi^{i}(u) \cdot f(u) d u \\
& =\int_{\mathcal{I}} f(u) d u .
\end{aligned}
$$

2.4. JMAP criterion. For fixed weighting functions, our goal is to jointly estimate both the spatially invariant PSF set $h^{i}, 1 \leq i \leq M$, and the image $f$ from the observation $g$. The probability distribution of $g$ conditionally to $h^{i}, 1 \leq i \leq M$, and $f$ is

$\operatorname{Pr}\left(g \mid f, h^{1}, \ldots, h^{M}\right)=\prod_{j \in \mathcal{I}_{d}} \frac{\left(\int_{R_{j}} \sum_{1 \leq i \leq M} h^{i} *\left(\psi^{i} \cdot f\right)(u) \mathrm{d} u+b_{g}\right)^{g(j)} e^{-\left(\int_{R_{j}} \sum_{1 \leq i \leq M} h^{i} *\left(\psi^{i} . f\right)(u) \mathrm{d} u+b_{g}\right)}}{g(j) !}$.

This inverse problem is ill-posed since the solution $\left(f, h^{1}, \ldots, h^{M}\right)$ is not unique. In particular, one trivial solution is $(g, \delta, \ldots, \delta)$; i.e., the space invariant PSFs are Dirac functions, and the recovered image is the same as the degraded image. It is thus important to regularize both the image and the PSFs. In this work, we propose estimating $\left(f, h^{1}, \ldots, h^{M}\right)$ by minimizing 
the regularized criterion

$$
\begin{aligned}
J\left(f, h^{1}, \ldots, h^{M}\right) & =\sum_{j \in \mathcal{I}_{d}}\left(\sum_{1 \leq i \leq M} \int_{R_{j}} h^{i} *\left(\psi^{i} \cdot f\right)(u) \mathrm{d} u+b_{g}\right) \\
& -\sum_{j \in \mathcal{I}_{d}} \mathrm{~g}(j) \cdot \log \left(\sum_{1 \leq i \leq M} \int_{R_{j}} h^{i} *\left(\psi^{i} \cdot f\right)(u) \mathrm{d} u+b_{g}\right) \\
& +\alpha \int_{\mathcal{I}}|D f|+\sum_{1 \leq i \leq M} \beta^{i}\left\|\nabla h^{i}\right\|_{2}^{2} .
\end{aligned}
$$

The two first terms correspond to the data fidelity component resulting from the antilog of (2.14). The third term is the total variation function [34] which allows us to smooth homogeneous areas of the recovered image while preserving sharp edges. The last term, introduced for regularizing the PSFs, allows us to promote the most spread out possible PSF and subsequently prevents a portion of the blur to be associated with the recovered image. $\alpha$ and $\beta^{i}, 1 \leq i \leq M$, are regularizing parameters that quantify the trade-off between these regularizing components and the data fidelity term. As the PSFs $h^{i}, 1 \leq i \leq M$, are different, the regularizing parameters $\beta^{i}, 1 \leq i \leq M$, could also be different. Parameter setting will be presented in section 4 . In addition to these regularizing terms, we take into account some other constraints in order to reduce the degree of freedom of this highly underdetermined problem. On the one hand, the positiveness and the normalization constraints are imposed on the space invariant PSFs. On the other hand, the positiveness and the flux conservation constraints are imposed on the image. According to Lemma 2.1, the constraints of flux conservation and the PSF normalization are redundant. However, both of them are imposed in the proposed estimation method since we use an alternating minimization procedure (see section 3 ).

Remark 1. In [8], we proposed a myopic restoration method in which the PSF is parametrized by a Gaussian function. This allows us to naturally impose positiveness and normalization constraints and also to reduce the number of unknown variables. However, this model strongly constrains the PSF shape and thus limits its applicability. Another limitation is that the dependency of the energy w.r.t. the PSF parameters is nonconvex. Thus the optimization w.r.t. these parameters leads to using time consuming algorithms such as simulated annealing. Advantages of the unparametrized PSF are both the high flexibility of the PSF shape and the convexity of the objective functional w.r.t. the PSF coefficients.

The problem we are interested in is formulated as follows:

$$
\left(\hat{f}, \hat{h}^{1}, \ldots, \hat{h^{M}}\right)=\arg \min _{\mathcal{F}_{c} \times \mathcal{H}^{M}} J\left(f, h^{1}, \ldots, h^{M}\right),
$$

where $c=\left\|g-b_{g}\right\|_{1}$, and $\mathcal{F}_{c}$ and $\mathcal{H}^{M}$ are as defined in subsection 2.2. Minimizing such a criterion is a difficult problem since it is not jointly convex w.r.t. $\left(f, h^{1}, \ldots, h^{M}\right)$. However, it is convex w.r.t. each variable separately.

One of the contributions of this work is the following theorem.

Theorem 2.4. Problem $\operatorname{Inf}_{\mathcal{F}_{c} \times \mathcal{H}^{M}} J\left(f, h^{1}, \ldots, h^{M}\right)$ has at least one solution. 
The proof of Theorem 2.4 is postponed to Appendix A. The proof remains valid also in the space invariant case. In fact, it suffices to set $M=1$ and $\psi^{1}(u)=1 \forall u \in \mathbb{R}^{n}$.

Remark 2. The solution of (2.16) is not unique. In fact, we can show that the criterion (2.15) is nonconvex w.r.t. all the variables $\left(f, h^{1}, \ldots, h^{M}\right)$ jointly. Nevertheless, it is convex w.r.t. each variable separately. Consequently, we can plan to use an alternating minimization algorithm.

3. Numerical minimization. We focus in this section on the numerical approximation of a solution of problem (2.16). For that, we consider the associated discrete functional. As the functional is convex w.r.t. each variable separately, we use an alternating minimization algorithm. Each minimization step reduces to the minimization of a criterion composed of Poisson and regularizing terms. A lot of algorithms have been proposed in the literature for that purpose $[18,36,23,17,44,11]$. In this work, we use a scale gradient projection (SGP) algorithm proposed in [11] for marginal minimizations. The main advantage of this gradient descent algorithm is to propose a scaling strategy and a step-length updating rule defined specifically for such a criterion in order to improve the speed of convergence. For the sake of compactness, we denote the global method SGPAM for scale gradient projection based alternating minimization. In this section, we first introduce the discrete version of the considered problem and present the alternating minimization scheme. Then, we introduce the SGP method in its general form. Finally, we show how we can apply this algorithm to the image and PSF estimation problems.

3.1. Discrete notation and alternating minimization scheme. We denote vectors by bold symbols and matrices by uppercase letters. The 2D or 3D image can thus be represented by an $N$-dimensional vector $\mathbf{f}=\left(f_{1}, \ldots, f_{N}\right)^{T} \in \mathbb{R}^{N}$ considering lexicographical order of pixels (or voxels), where $N$ is the image size. The space invariant blur operator can be represented by a matrix $H \in \mathbb{R}^{N \times N}$ which is block circulant with circulant blocks for 2 D images and block circulant with circulant blocks of circulant blocks for 3D images. The circular convolution is denoted by $H \mathbf{f}$. Roles can be inverted, and the circular convolution can be written as $F \mathbf{h}$, where $F \in \mathbb{R}^{N \times N}$ is a matrix representing the image and $\mathbf{h} \in \mathbb{R}^{N}$ is an $N$-dimensional vector representing the space invariant PSF. Considering that the elementary regions are of equal size (i.e., $\left|R_{j}\right|=1 \forall j \in \mathcal{I}^{d}$ ), the discrete function we are interested in is expressed as

$$
\begin{aligned}
J\left(\mathbf{f}, \mathbf{h}^{1}, \ldots, \mathbf{h}^{M}\right) & =\mathbf{1}^{T}\left(\sum_{1 \leq i \leq M} H^{i} \psi^{i} \mathbf{f}+\mathbf{b}_{\mathbf{g}}\right)-\mathbf{g}^{T} \log \left(\sum_{1 \leq i \leq M} H^{i} \psi^{i} \mathbf{f}+\mathbf{b}_{\mathbf{g}}\right) \\
& +\alpha\|\nabla \mathbf{f}\|_{1}+\sum_{1 \leq i \leq M} \beta^{i}\left\|\nabla \mathbf{h}^{i}\right\|_{2}^{2},
\end{aligned}
$$

where $\mathbf{1} \in \mathbb{R}^{N}$ stands for the $N$-size vector whose components are all equal to 1 , and $\mathbf{b}_{\mathbf{g}} \in \mathbb{R}^{N}$ is a strictly positive constant vector (all its components are equal) modeling the background noise. Functions $\psi^{i} \in \mathbb{R}^{N \times N}, 1 \leq i \leq M$, are diagonal matrices referring to weighting coefficients for the space variant blur, and $H^{i} \in \mathbb{R}^{N \times N}, 1 \leq i \leq M$, are matrices modeling the space invariant blur operators. Therefore the sum over $j \in \mathcal{I}_{d}$ is replaced by the scalar products $1^{T}$. () and $g^{T}$.( ). Numerical computations of the gradients as well as $l_{1}$ and $l_{2}$ norms are given in Appendix B. 
The constrained optimization problem we propose to solve is

$$
\begin{aligned}
\left(\hat{\mathbf{f}}, \hat{\mathbf{h}}^{1}, \ldots, \hat{\mathbf{h}}^{M}\right)=\quad \arg \min _{\left(\mathbf{f}, \mathbf{h}^{1}, \ldots, \mathbf{h}^{M}\right)} J\left(\mathbf{f}, \mathbf{h}^{1}, \ldots, \mathbf{h}^{M}\right) \\
\text { subject to }\left\{\begin{array}{l}
\mathbf{f} \geq 0, \\
\|\mathbf{f}\|_{1}=c, \\
\mathbf{h}^{i} \geq 0,1 \leq i \leq M, \\
\left\|\mathbf{h}^{i}\right\|_{1}=1,1 \leq i \leq M, \\
\operatorname{supp}\left(\mathbf{h}^{i}\right) \subset B, 1 \leq i \leq M,
\end{array}\right.
\end{aligned}
$$

where $c=\left\|\mathrm{g}-\mathrm{b}_{\mathrm{g}}\right\|_{1}$ is a positive constant and $B$ is a given set corresponding to the support of the space invariant PSFs. As shown in Lemma 2.1, constraints $\left\|\mathbf{h}^{i}\right\|_{1}=1$ and $\|\mathbf{f}\|_{1}=c$ are redundant. However, it is important to incorporate both since we use an alternating optimization algorithm which does not guarantee that the two constraints are simultaneously satisfied (see subsection 3.2). One trivial way for minimizing $J$ is to stack the image and the PSF vectors into the same vector $X$ and then apply any optimization method on $X$. However, this is not the optimal way for dealing with such a multivariate optimization problem. In fact, this is extremely slow since the gradients of the criterion w.r.t. the image and w.r.t. the PSF vectors may have different orders of magnitude. An alternating minimization scheme is known to be appropriate to such a situation; i.e., the objective function to be minimized has two or more unknowns with different orders of magnitude. It consists in splitting the problem into two or more stages and alternating between them. First, keeping the PSF vectors constant, estimate the image. Then, update each of the space invariant PSFs sequentially by fixing the remaining PSFs and the image to their previous estimates. That is, starting from an initial guess of the PSFs $\hat{\mathbf{h}}^{(0)}, \ldots, \hat{\mathbf{h}}^{\hat{M}^{(0)}}$, the algorithm is given by the following two steps:

- Image estimation step:

$$
\begin{array}{r}
\hat{\mathbf{f}}^{(k+1)}=\arg \min _{f} J\left(\mathbf{f}, \hat{\mathbf{h}}^{(k)}, \ldots, \hat{\mathbf{h}}^{\left(\hat{M}^{(k)}\right.}\right) \\
\text { subject to }\left\{\begin{array}{l}
\mathbf{f} \geq 0, \\
\sum_{1 \leq i \leq N} f_{i}=c .
\end{array}\right.
\end{array}
$$

- PSF estimation step:

$$
\begin{aligned}
& \hat{\mathbf{h}}^{(k+1)}=\arg \min _{\mathbf{h}^{1}} J\left(\hat{\mathbf{f}}^{(k+1)}, \mathbf{h}^{1}, \hat{\mathbf{h}}^{(k)}, \ldots, \hat{\mathbf{h}}^{\hat{M}^{(k)}}\right) \\
& \text { subject to }\left\{\begin{array}{l}
\mathbf{h}^{1} \geq 0, \\
\sum_{1 \leq i \leq N} h_{i}^{1}=1, \\
\operatorname{supp}\left(\mathbf{h}^{1}\right) \subset B
\end{array}\right.
\end{aligned}
$$




$$
\begin{aligned}
\hat{\mathbf{h}}^{\hat{M}^{(k+1)}=\arg \min _{\mathbf{h}^{M}} J\left(\hat{\mathbf{f}}^{(k+1)}, \hat{\mathbf{h}}^{1^{(k+1)}}, \ldots, \mathbf{h}^{\hat{M}-1}{ }^{(k+1)}, \mathbf{h}^{M}\right)} \\
\text { subject to }\left\{\begin{array}{l}
\mathbf{h}^{M} \geq 0, \\
\sum_{1 \leq i \leq N} h_{i}^{M}=1, \\
\operatorname{supp}\left(\mathbf{h}^{M}\right) \subset B .
\end{array}\right.
\end{aligned}
$$

We show in the following subsections how to solve these problems by using the SGP algorithm. Thanks to this algorithm, the global energy decreases at each step of the alternating minimization algorithm:

$$
\begin{array}{r}
J\left(\hat{\mathbf{f}}^{(0)}, \hat{\mathbf{h}}^{(0)}, \ldots, \hat{\mathbf{h}}^{(0)}\right) \geq J\left(\hat{\mathbf{f}}^{(1)}, \hat{\mathbf{h}}^{(0)}, \ldots, \hat{\mathbf{h}}^{(0)}\right) \geq J\left(\hat{\mathbf{f}}^{(1)}, \hat{\mathbf{h}}^{(1)}, \ldots, \hat{\mathbf{h}}^{(0)}\right) \\
\geq \cdots \geq J\left(\hat{\mathbf{f}}^{(1)}, \hat{\mathbf{h}}^{(1)}, \ldots, \hat{\mathbf{h}}^{\left(\hat{M}^{(1)}\right.}\right) \geq J\left(\hat{\mathbf{f}}^{(2)}, \hat{\mathbf{h}}^{\left({ }^{(1)}\right.}, \ldots, \hat{\mathbf{h}}^{\hat{M}^{(1)}}\right) \geq \cdots \geq J\left(\hat{\mathbf{f}}^{(2)}, \hat{\mathbf{h}}^{(2)}, \ldots, \hat{\mathbf{h}}^{(2)}\right) .
\end{array}
$$

Hence we have

$$
J\left(\hat{\mathbf{f}}^{(k)}, \hat{\mathbf{h}}^{(k)}, \ldots, \hat{\mathbf{h}}^{(k)}\right) \geq J\left(\hat{\mathbf{f}}^{(k+1)}, \hat{\mathbf{h}}^{(k+1)}, \ldots, \hat{\mathbf{h}}^{(k+1)}\right) \quad \forall k .
$$

Hence, the sequence $\left(J\left(\hat{\mathbf{f}}^{(k)}, \hat{\mathbf{h}}^{1^{(k)}}, \ldots, \hat{\mathbf{h}}^{\hat{M}^{(k)}}\right)\right)_{k \in \mathbb{N}}$ is decreasing and bounded below, so it is convergent.

Remark 3.

- In the discrete setting, it is obvious that the sequences $\hat{\mathbf{f}}^{(k)}$ and $\hat{\mathbf{h}}^{i}{ }^{(k)}$ are bounded. So we can extract convergent subsequences. As we will use a smooth approximation of total variation and as the background constant $b_{g}$ is nonzero, the global energy is differentiable. Thus we can apply the result of paper [10] and deduce that the previous subsequences converge towards a stationary point of the constrained problem (3.2) (see also [14] for classical and new results on this type of problem).

- It would be interesting to study the relationship between the solution in the discrete setting and the one in the continuous setting when the mesh size tends to zero. This could be obtained by using $\Gamma$-convergence tools, but it is beyond the scope of this paper and will be studied in a forthcoming paper.

3.2. SGP algorithm. Several algorithms have been proposed in the literature for minimizing criteria composed of a data term proportional to the Kullback-Leibler distance between measured data and the blurred model (due to Poissonian statistics) plus a regularization term such as the ones in (3.3), (3.4), and (3.5). As we use a smooth approximation of total variation (see Appendix B), the minimization problems turn out to be convex and differentiable. In image processing, algorithms based on the proximal method or the alternating direction method of multipliers (ADMM) are extensively used [17, 44, 11, 18, 13, 36, 23]. In the alternating minimization procedure, such optimization algorithms are necessarily running a lot of time. Thus we choose the SGP algorithm described in [11] since this gradient-type algorithm has been computationally optimized for this kind of energy. Moreover, it is proven in [10] that the global inexact block coordinate minimization process using the SGP algorithm converges toward a stationary point of functional (2.15). 
We limit ourselves to the presentation of the main lines of this method. Details of this algorithm can be found in [11]. For a given generic problem of the form

$$
\min _{\mathbf{x} \in \Omega} \mathbf{J}(\mathbf{x})
$$

where $\mathbf{x}=\left(x_{1}, \ldots, x_{N}\right)^{T} \in \mathbb{R}^{N}$ is an $N$-dimensional vector, $\Omega \subset \mathbb{R}^{N}$ is a closed convex set, and $\mathbf{J}: \Omega \rightarrow \mathbb{R}$ is a differentiable function, the SGP algorithm approximates the fixed point

$$
\mathbf{x}^{*}=P_{\Omega, S}\left(\mathbf{x}^{*}-\delta S \nabla \mathbf{J}\left(\mathbf{x}^{*}\right)\right),
$$

where $\delta$ is a positive scalar referring to the step-length of the proposed descent method and $S$ is a symmetric positive definite $N \times N$ matrix which corresponds to the scaling matrix.

$P_{\Omega, S}: \mathbb{R}^{N} \rightarrow \Omega$ used in (3.8) is the projection operator

$$
P_{\Omega, S}(\mathbf{x})=\arg \min _{\mathbf{y} \in \Omega}\|\mathbf{y}-\mathbf{x}\|_{S},
$$

where $\|\cdot\|_{S}$ indicates the vector norm associated with the symmetric positive definite matrix $S\left(\|\mathbf{x}\|_{S}=\sqrt{\mathbf{x}^{T} S \mathbf{x}}\right)$.

$(\delta S)$ used in (3.8) allows one to approximate the inverse of the Hessian matrix of $\mathbf{J}$ in order to enforce quasi-Newton properties and thus provide a good convergence rate (see, for example, [6] for a quasi-Newton splitting method). Appropriate selection of these two latter parameters, i.e., the scaling matrix $S$ and the step-length $\delta$, is proposed in [47]. The scaling matrix $S$ is chosen to give better convergence than classical gradient search methods (see subsections 3.3 and 3.4), and the step-length $\delta$ is chosen by using an adaptive alternative of Barzilai and Borwein rules $[5,11,19]$. Note that the convergence of the proposed method is proved in [11] when the elements $\left(s_{i j}\right)_{1 \leq i, j \leq N}$ of $S$ verify

$$
\frac{1}{L} \leq s_{i j} \leq L, L>1 \text {. }
$$

Sequences $\hat{\mathbf{f}}^{(k)}$ and $\hat{\mathbf{h}}^{(k)}$ are bounded in $\mathbb{R}^{N}$; then up to a subsequence they converge to $\hat{\mathbf{f}}^{*}$ and $\hat{\mathbf{h}}^{i}$. From results of Bonettini in $[10],\left(\hat{\mathbf{f}}^{*}, \hat{\mathbf{h}}^{1^{*}}, \ldots, \hat{\mathbf{h}}^{M}\right)$ is a stationary point for the constrained problem (3.2).

To apply the SGP method for image and PSF estimations, it suffices to specify the objective function $\mathbf{J}$, its derivative $\nabla \mathbf{J}$, the scaling matrix $S$, and the step-length $\delta$ for the considered problems; the rest of the algorithm is as described in [11]. This will be the goal of the following two subsections.

3.3. SGP algorithm for image estimation. Consider the optimization problem (3.3). The PSF set being fixed, the objective function to be minimized is reduced to

$$
\mathbf{J}_{0}(\mathbf{f})=\mathbf{1}^{T}\left(\sum_{1 \leq i \leq M} H^{i} \psi^{i} \mathbf{f}+\mathbf{b}_{\mathbf{g}}\right)-\mathbf{g}^{T} \log \left(\sum_{1 \leq i \leq M} H^{i} \psi^{i} \mathbf{f}+\mathbf{b}_{\mathbf{g}}\right)+\alpha\|\nabla \mathbf{f}\|_{1}
$$

where $H^{i}$ models the convolution with the PSF $\mathbf{h}^{i}$. We apply on this functional the SGP algorithm with the positiveness and flux conservation constraints on the variable $\mathbf{f}$. For that, 
we need to express the gradient of the functional $\nabla \mathbf{J}_{0}(\mathbf{f})$ and the scaling matrix $S$ used in (3.8). The step-length $\delta$ can be derived from $S$ using equations (29) and (30) of [11]. Note that the considered objective function differs from that used in [11] since we use a regularizing term and a space variant blur operator. It also differs from that used in [47] by using a space variant blur operator.

Now, we give the expression of $\nabla \mathbf{J}_{0}(\mathbf{f})$ :

$$
\nabla \mathbf{J}_{0}(\mathbf{f})=\sum_{1 \leq i \leq M} \psi^{i} H^{i^{T}}\left(\mathbf{1}-Y^{-1} \mathbf{g}\right)+\alpha \nabla \mathbf{J}_{0}^{R}(\mathbf{f}),
$$

where $Y$ is a diagonal matrix with the following entries: $Y=\sum_{1 \leq i \leq M} H^{i} \psi^{i} \mathbf{f}+\mathbf{b}_{\mathbf{g}}$. As the PSFs are normalized, we have $\sum_{1 \leq i \leq M} \psi^{i} H^{i^{T}} \mathbf{1}=\mathbf{1}$, which allows us to write $\nabla \mathbf{J}_{0}$ as follows:

$$
\nabla \mathbf{J}_{0}(\mathbf{f})=\mathbf{1}-\sum_{1 \leq i \leq M} \psi^{i} H^{i^{T}} Y^{-1} \mathbf{g}+\alpha \nabla \mathbf{J}_{0}^{R}(\mathbf{f})
$$

The gradient of the regularization term $\nabla \mathbf{J}_{0}^{R}(\mathbf{f})$ can be split into positive and negative parts:

$$
-\nabla \mathbf{J}_{0}^{R}(\mathbf{f})=U_{0}^{R}(\mathbf{f})-V_{0}^{R}(\mathbf{f}),
$$

where $V_{0}^{R}(\mathbf{f})$ and $U_{0}^{R}(\mathbf{f})$ are nonnegative vectors in $\mathbb{R}^{N}$ given in Appendix B. Thus $\nabla \mathbf{J}_{0}$ writes as

$$
-\nabla \mathbf{J}_{0}(\mathbf{f})=\left(\sum_{1 \leq i \leq M} \psi^{i} H^{i^{T}} Y^{-1} \mathbf{g}+\alpha U_{0}^{R}(\mathbf{f})\right)-\left(\mathbf{1}+\alpha V_{0}^{R}(\mathbf{f})\right) .
$$

The minimum $\mathbf{f}^{*}$ of function (3.11) verifies the following condition: $\mathbf{f}^{*} \nabla \mathbf{J}_{0}\left(\mathbf{f}^{*}\right)=0$, which corresponds to the first Karush-Kuhn-Tucker (KKT) conditions of problem (3.3) [27]. That is, one can use the following minimizing iteration:

$$
\mathbf{f}^{(k+1)}=Z^{(k)^{-1}} \mathbf{f}^{(k)}\left(\sum_{1 \leq i \leq M} \psi^{i} H^{i^{T}} Y^{(k)^{-1}} \mathbf{g}+\alpha U_{0}^{R}\left(\mathbf{f}^{(k)}\right)\right),
$$

where $Z^{(k)}$ is a diagonal matrix with entries $\mathbf{1}+\alpha V_{0}^{R}\left(\mathbf{f}^{(k)}\right)$.

Remark that the above equation can be seen as a regularized version of the RichardsonLucy (RL) or maximum likelihood expectation maximization (MLEM) algorithm [37] fitted to a space variant PSF. Note that this version has an important advantage w.r.t. the widely used Richardson-Lucy total variation (RLTV) algorithm [16]. Thanks to the proposed decomposition of the gradient of the regularization term, the algorithm preserves the positiveness of the solution which is not guaranteed by the RLTV algorithm. Furthermore, we can prove that (3.16) can be expressed as

$$
\mathbf{f}^{(k+1)}=\mathbf{f}^{(k)}-Z^{(k)^{-1}} \mathbf{f}^{(k)} \nabla \mathbf{J}_{0}\left(\mathbf{f}^{(k)}\right) .
$$


This iteration corresponds to the SGP algorithm for $\delta^{(k)}=1$ and $S^{(k)}$ a diagonal matrix defined by

$$
S^{(k)}=\operatorname{diag}\left(\mathbf{s}^{(k)}\right), \mathbf{s}^{(k)}=\left(s_{i}{ }^{(k)}\right)_{1 \leq i \leq N}=Z^{(k)^{-1}} \mathbf{f}^{(k)} .
$$

This expression of the scaling matrix leads to a better convergence than (3.17) thanks to the adaptive selection of the step-length as in [11]. Note that it is possible to further optimize the choice of $S$, for example, by studying the optimal choice of the decomposition of $\nabla \mathbf{J}_{0}^{R}$. Following [11], the scaling matrix is chosen as

$$
s_{i}{ }^{(k)}=\min \left\{L, \max \left\{\frac{1}{L}, s_{i}{ }^{(k)}\right\}\right\}, i=1, \ldots, N, L>1,
$$

with $L$ fixed to a high value (e.g., $L=10^{10}$ in our tests). By computing $\nabla \mathbf{J}_{0}(\mathbf{f})$ as in (3.15) and choosing $S$ as in (3.18) and (3.19), one can easily apply the SGP algorithm on $\mathbf{f}$.

3.4. SGP algorithm for PSF estimation. Now, to solve problems (3.4) and (3.5), we follow a similar strategy as previously. Consider, for example, the optimization problem w.r.t. the PSF $\mathbf{h}^{j}, j \in\{1, \ldots, M\}$. Denoting by $F^{i}, i \in\{1, \ldots, M\}$, the matrix obtained from the vector $\psi^{i} \mathbf{f}$, we look for $\mathbf{h}^{j}, j \in\{1, \ldots, M\}$, minimizing

$$
\mathbf{J}_{j}\left(\mathbf{h}^{j}\right)=\mathbf{1}^{T}\left(\sum_{1 \leq i \leq M} F^{i} \mathbf{h}^{i}+\mathbf{b}_{\mathbf{g}}\right)-\mathbf{g}^{T} \log \left(\sum_{1 \leq i \leq M} F^{i} \mathbf{h}^{i}+\mathbf{b}_{\mathbf{g}}\right)+\beta^{j} \mathbf{J}_{j}^{R}\left(\mathbf{h}^{j}\right),
$$

with $\mathbf{J}_{j}^{R}\left(\mathbf{h}^{j}\right)=\left\|\nabla \mathbf{h}^{j}\right\|_{2}^{2}$. To apply the SGP algorithm on the above functional with positiveness, PSF normalization, and PSF support constraints, we need to compute $\nabla \mathbf{J}_{j}\left(\mathbf{h}^{j}\right)$ and $S$ for this problem. Note that the SGP algorithm has never been used for such a problem where the regularizing term is quadratic and the operator applied on the variable to be estimated is not normalized $\left(F^{i} \mathbf{1} \neq \mathbf{1}\right)$.

Let us first express the gradient of functional $\mathbf{J}_{j}^{R}\left(\mathbf{h}^{j}\right)$ w.r.t. $\mathbf{h}^{j}$ :

$$
\nabla \mathbf{J}_{j}\left(\mathbf{h}^{j}\right)=F^{j^{T}}\left(\mathbf{1}-W^{-1} \mathbf{g}\right)+\beta^{j} \nabla \mathbf{J}_{j}^{R}\left(\mathbf{h}^{j}\right),
$$

with $W=\operatorname{diag}\left(\sum_{1 \leq i \leq M} F^{i} \mathbf{h}^{i}+\mathbf{b}_{\mathbf{g}}\right)$ a diagonal matrix. Analogously as in (3.14), we decompose $\nabla \mathbf{J}_{j}^{R}\left(\mathbf{h}^{j}\right)$ into positive and negative parts (see Appendix B):

$$
-\nabla \mathbf{J}_{j}^{R}\left(\mathbf{h}^{j}\right)=U_{j}^{R}\left(\mathbf{h}^{j}\right)-V_{j}^{R}\left(\mathbf{h}^{j}\right) .
$$

Now, we can derive an expression of $S$ from the following iteration of the regularized RL algorithm:

$$
\mathbf{h}^{j^{(k+1)}}=E^{(k)^{-1}} \mathbf{h}^{j(k)}\left(F^{j^{T}} W^{(k)^{-1}} \mathbf{g}+\beta^{j} U_{j}^{R}\left(\mathbf{h}^{j^{(k)}}\right)\right),
$$

where

$$
\begin{aligned}
& E^{(k)}=\operatorname{diag}\left(F^{j} \mathbf{1}+\beta^{j} V_{j}^{R}\left(\mathbf{h}^{j^{(k)}}\right)\right), \\
& W^{(k)}=\operatorname{diag}\left(F^{j} \mathbf{h}^{j^{(k)}}+\sum_{i \in\{1, \ldots, M\} \backslash\{j\}} F^{i} \mathbf{h}^{i}+\mathbf{b}_{\mathbf{g}}\right) .
\end{aligned}
$$


We can prove that (3.23) is equivalent to

$$
\mathbf{h}^{j^{(k+1)}}=\mathbf{h}^{j^{(k)}}-E^{(k)^{-1}} \mathbf{h}^{j(k)} \nabla \mathbf{J}_{j}\left(\mathbf{h}^{j}\right) .
$$

Consequently, $S^{(k)}$ is given by $S^{(k)}=\operatorname{diag}\left(E^{(k)^{-1}} \mathbf{h}^{j^{(k)}}\right)$.

4. Experimental tests. We test the method on two kinds of data: 2D images of a digital camera and 3D images of confocal microscopy. Different parameters of the proposed algorithm need to be adjusted. First, the weighting functions are set a priori according to the considered application. In our experiment linear weighting functions are considered. In fact, they are shown to give a precision similar to the one obtained by the weighting functions estimated by a principal component analysis (PCA) method $[1,46]$. Second, the SGP parameters are set as in [11]: $L=10^{10}, \delta_{\min }=10^{-5}, \delta_{\max }=10^{5}$. The global algorithm converges to a stationary point whatever the number of iterations of the elementary minimization algorithm (SGP). Thus, we fix the number of iterations of the SGP algorithm to 10 and stop the global algorithm when the global energy remains unchanged (i.e., the normalized energy error between two successive iterations is less than $10^{-4}$ ) or when the number of the iterations reaches 1000 . For fixed parameters of this algorithm, we set the regularization parameters $\alpha$ and $\beta_{i}$ by running the algorithm several times, for different values of $\alpha, \beta_{i}$, and selecting those that optimize a quality measure [49]. In fact, these regularization parameters have antagonist effects on the PSFs and the image: a high value of $\beta_{i}$ leads to an excessively large PSF and ringing artifacts in the recovered image. A high value of $\alpha$ leads to the staircasing effect in the reconstructed image. In our experiments, a set of possible parameter values are proposed by the user; the optimal parameters are those that maximize a no-reference quality measure proposed in [49]. The advantage of this measure is that it gives a relative score indicating the levels of blur and the noise level whatever the type of image. We exploit the behavior of this measure which decreases for a given image when increasing the blur and/or the noise. This method requires running the estimation program for each possible parameter set and thus is time consuming. Let us remark that it is the case for most parameter estimation methods, even more so when there is a set of unknown parameters (more than one), which is the case in this work.

Our program is implemented on MATLAB and tested on a machine having a processor frequency of $1.86 \mathrm{GHz}$. In order to assess the performance of the proposed method, we use two different evaluation criteria:

- The relative reconstruction error is defined as

$$
R R E(\mathbf{f}, \hat{\mathbf{f}})=\frac{\|\hat{\mathbf{f}}-\mathbf{f}\|_{2}}{\|\mathbf{f}\|_{2}}
$$

where $\mathbf{f}$ refers to the true image vector and $\hat{\mathbf{f}}$ is an estimate of the latter.

- The structural similarity index (SSIM) allowing us to detect changes in structures between two images [42] is given by

$$
\operatorname{SSIM}(\mathbf{f}, \hat{\mathbf{f}})=\frac{\left(2 \mu_{\hat{\mathbf{f}}} \mu_{\mathbf{f}}+c_{1}\right)\left(2 \sigma_{\hat{\mathbf{f}} \mathbf{f}}+c_{2}\right)}{\left(\mu_{\hat{\mathbf{f}}}^{2} \mu_{\mathbf{f}}^{2}+c_{1}\right)\left(\sigma_{\hat{\mathbf{f}}}^{2} \sigma_{\mathbf{f}}^{2}+c_{2}\right)},
$$




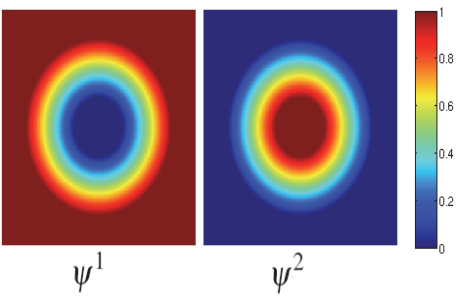

(a)

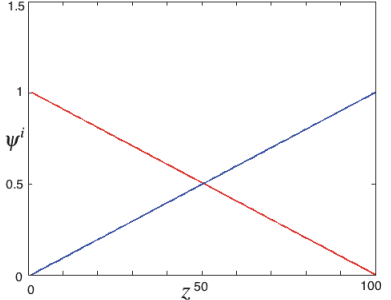

(b)

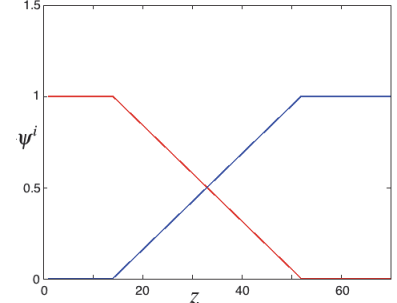

(c)

Figure 1. (a) Circular variations of two considered weighting functions for the test image A: $\psi^{1}$ is increasing from the center to the edges of the image, while $\psi^{2}$ is decreasing from the center to the edges of the image; the presented image intensities vary from 0 in blue to 1 in red. Similar circular varying weighting functions are used for the test image B. (b) and (c) Linear variation of the two considered weighting functions along the $z$-axis for the test images $C$ and $D$ : the presented plots show only a z-line of the $3 D$ weighting functions.

where $\mu_{\mathbf{f}}$ and $\mu_{\hat{\mathbf{f}}}$ are, respectively, averages of $\mathbf{f}$ and $\hat{\mathbf{f}} ; \sigma_{\mathbf{f}}^{2}$ and ${\sigma_{\hat{\mathbf{f}}}}^{2}$ are their variances; and $\sigma_{\hat{\mathbf{f}} \mathbf{f}}$ is the covariance of $\mathbf{f}$ and $\hat{\mathbf{f}}$. $c_{1}$ and $c_{2}$ are two constants for stabilizing the division. We can choose $c_{1}=\left(k_{1} R\right)^{2}, c_{2}=\left(k_{2} R\right)^{2}$, with $R$ the dynamic range of the pixel-values, and $k_{1}$ and $k_{2}$ two constants that can be set as $k_{1}=0.01, k_{2}=0.03$. The closer the SSIM value is to 1 , the more the image structures are similar.

We compare the SGPAM method with another alternating minimization based algorithm where elementary minimizations are performed using a regularized RL algorithm (see [16]). We denote the latter method by RRLAM. In order to show the interest of the proposed space variant blur model, we present some blind deconvolution (BD) results using a stationary PSF (only one space invariant PSF).

4.1. Test on 2D digital camera images. The first experiment is related to optical distortions in a digital camera which are not the same at the center and at the edges of the image due to the difference of thickness of the spherical lenses at the center and at the edges $[12,38]$. That is why we consider in our test circular variations of the PSF from the center to the edges of the image. In Figure 1(a), we show an example of two circular varying weighting functions. The dominant noise in a digital camera is Gaussian due to the high illumination. Nevertheless, our method designed for Poisson observed images can be applied. In fact, for high intensity levels, the Poisson distribution tends to a Gaussian distribution; cf. [39]. Our tests are performed on two different simulated images of a digital camera, with different blur variations and different noise levels.

\subsubsection{First test.}

Image simulation. Consider the sharp image of $256 \times 256$ pixels, depicted in Figure 2(a), that we denote by the letter "A." We blur this image with a space variant PSF constructed from a combination of two Gaussian PSFs displayed in Figures 2(e) and (i) in a logarithmic scale. For that, we use two circular varying weighting functions as in Figure 1(a). We add to this blurred image a background constant of $b_{g}=10^{-2}$, resulting in an image $z$. Then we compute in each pixel $i$ a realization of a Poisson random variable of parameter $z_{i}$. The final degraded image, depicted in Figure 2(b), has a peak signal to noise ratio (PSNR) of $18 d B$. 


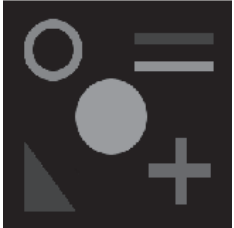

(a)

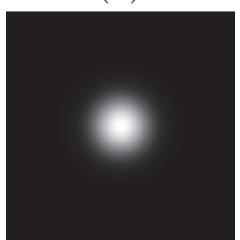

(e)

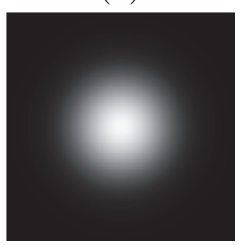

(i)

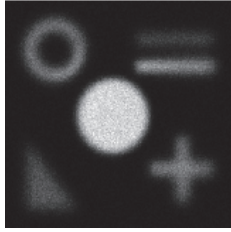

(b)

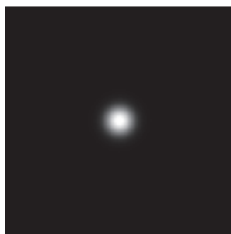

(f)

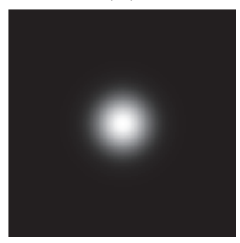

(j)

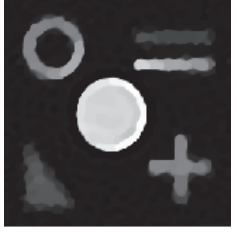

(c)

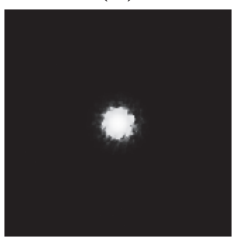

(g)

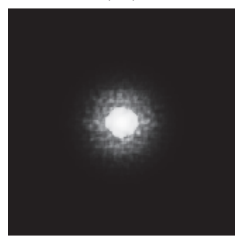

(k)

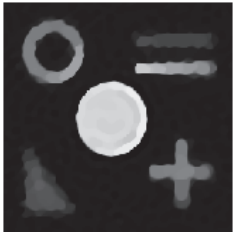

(d)

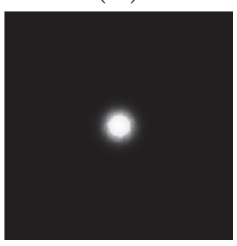

(h)

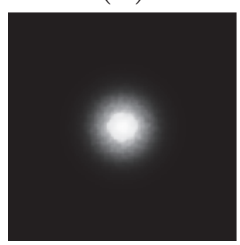

(1)

Figure 2. Blind restoration results on the test image A: (a) original image, (b) observation, (c) restoration using SGPAM, (d) restoration using RRLAM, (e) first true PSF $h^{1}$, (f) initial PSF ${\hat{h^{1}}}^{(0)}$, (g) estimated ${\hat{h^{1}}}^{1}$ with SGPAM, (h) estimated $\hat{h}^{1}$ with RRLAM, (i) second true PSF $h^{2}$, (j) initial PSF ${\hat{h^{2}}}^{(0)},(\mathrm{k})$ estimated $\hat{h}^{2}$ with SGPAM, (1) estimated $\hat{h}^{2}$ with RRLAM ( $\alpha=0.005$ and $\left.\beta^{i}=10^{3}, i=1,2\right)$.

\section{Table 1}

SGPAM method vs. RRLAM method for space variant (SV) and space invariant (SI) PSFs. Tests are presented for simulated $2 D$ images of a digital camera. $t_{m}$ is the mean computing time per iteration, and $t_{g}$ is the global computing time.

\begin{tabular}{|l|c|r|r|r|r|r|r|r|}
\hline & \multicolumn{4}{|c|}{ Image A } & \multicolumn{4}{c|}{ Image B } \\
\hline & \multicolumn{2}{|c|}{ RRLAM } & \multicolumn{2}{|c|}{ SGPAM } & \multicolumn{2}{c|}{ RRLAM } & \multicolumn{2}{c|}{ SGPAM } \\
\hline & SI & SV & \multicolumn{1}{c|}{ SI } & SV & SI & SV & SI & SV \\
\hline RRE (\%) & 8.71 & 6.18 & 7.62 & 6.31 & 2.69 & 1.75 & 2.52 & 1.73 \\
\hline SSIM & 0.933 & 0.955 & 0.943 & 0.955 & 0.934 & 0.963 & 0.930 & 0.962 \\
\hline$t_{m}(\mathrm{~min})$ & 0.010 & 0.01 & 0.011 & 0.02 & 0.08 & 0.23 & 0.22 & 0.43 \\
\hline$t_{g}(\mathrm{~min})$ & 10.59 & 18.01 & 6.31 & 18.97 & 6.48 & 16.49 & 5.57 & 13.82 \\
\hline nb it & 1000 & 1000 & 563 & 796 & 79 & 71 & 25 & 31 \\
\hline
\end{tabular}

Test and comparison with RRLAM. Starting from a random initial guess of the two PSFs, which is not very far from the true ones (cf. Figures $2(\mathrm{f})$ and $(\mathrm{j})$ ), we run the SGPAM algorithm on that image. We compare this result to that obtained by the RRLAM algorithm (Figures 2(d), (h), and (l)). Constraints of positiveness, flux conservation, and PSF normalization are added to that algorithm by projecting each of the estimates on a convenient set. In Table 1 , we give the RRE and the SSIM measures, the mean computing time per iteration $\left(t_{m}\right)$, the global computing time $\left(t_{g}\right)$, and the required number of iterations (nb it). For this test, RRLAM and SGPAM show comparable results. 


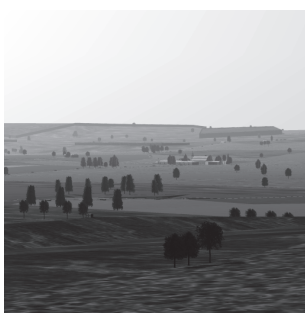

(a)

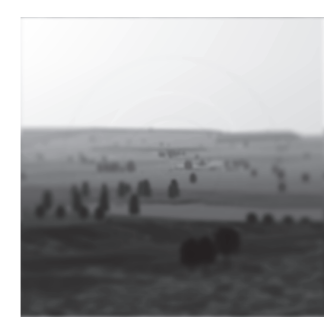

(b)

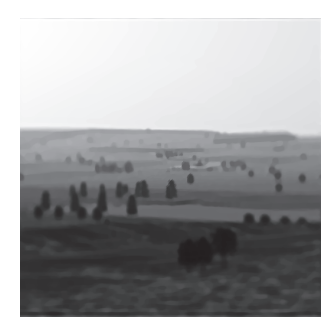

(c)

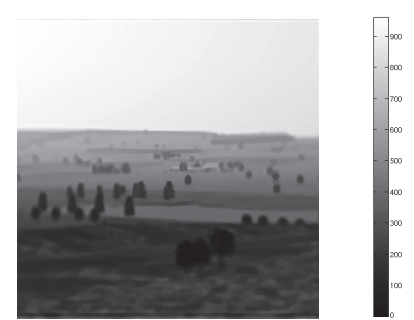

(d)

Figure 3. Blind restoration results on the test image $B$ provided by ATE (used with permission): (a) original image, (b) degraded image, (c) restoration using SGPAM, (d) restoration using RRLAM $\left(\alpha=10^{-3}\right.$ and $\left.\beta^{i}=10^{-4}, i=1, \ldots, 3\right)$.

Comparison with $B D$. We compare our result with those obtained by considering one single space invariant PSF. Numerical results presented in Table 1 show the interest of the space variant blur model w.r.t. the space invariant one.

\subsubsection{Second test.}

Image simulation. Our second test is performed on another simulated image with a more realistic blur model. In fact, in the previous test, the PSFs used are Gaussian, while in this test we use real PSFs of a digital camera system. Consider the original image of Figure 3(a) of size $500 \times 500$ pixels. We denote this test image by letter "B." We blurred this image using three space invariant PSFs and thre circular weighting functions (cf. Figure 1(a)). Then a background constant of $b_{g}=10^{-5}$ is added and a Poisson noise degradation is introduced, leading to the image depicted in Figure 3(b) with a PSNR of $29 \mathrm{~dB}$.

Test and comparison with RRLAM. Restoration results using SGPAM and RRLAM, respectively, are depicted in Figures 3(c) and (d). Evaluation criteria and computing time given in Table 1 show that SGPAM gives practically the same result as RRLAM but with a lower computational time.

Comparison with $B D$. One can remark from the evaluation criteria of Table 1 the interest of the proposed space variant restoration approach w.r.t. BD.

4.2. Test on 3D confocal microscopy images. Confocal laser scanning microscopy (CLSM) [26, 31] allows one to observe 3D biological living specimens at a resolution of a few hundred nanometers. However, 3D images acquired with this system are distorted by a blur varying along the depth, i.e., the $z$-axis due to a light refraction phenomenon when crossing mediums of different refractive indexes. The PSF of this system is $3 \mathrm{D}$ and different for each point along the $z$-axis. Consequently, we deal with $3 \mathrm{D}$ image restoration. The PSF variation has mainly three particularities when increasing the depth: radial and axial spread of the 3D PSF, axial dissymmetry, and axial shift of the main lobe w.r.t. the central plane (see Figures 4(e) and (i), which show axial slices of two PSFs at $0 \mu \mathrm{m}$ and $14.5 \mu \mathrm{m}$ of depths, respectively). Knowing all the imaging parameters, such as light wavelength and refractive indexes of the different system mediums, the PSF at different depths can be computed using the theoretical model developed in [20,21]. Nevertheless, some of these parameters are inaccessible, especially the refractive index, which is very sensitive to changes of the temperature. That is why a blind or semiblind restoration method needs to be developed for this system. 


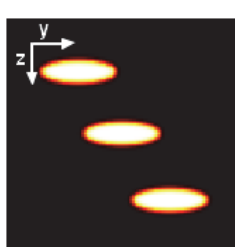

(a)

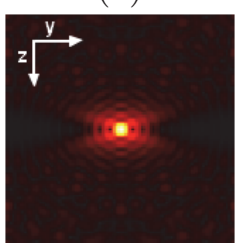

(e)

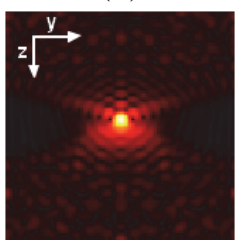

(i)

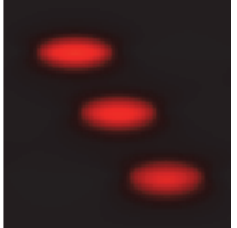

(b)

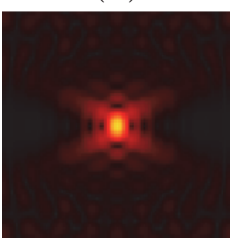

(f)

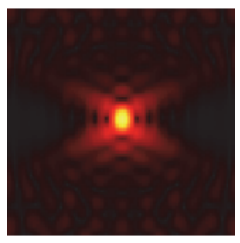

(j)

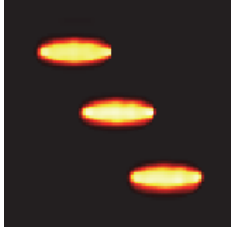

(c)

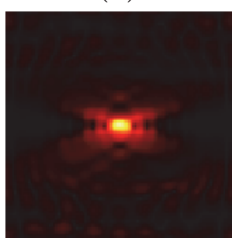

(g)

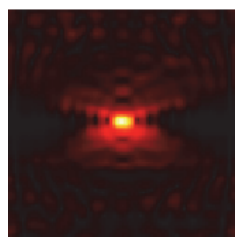

(k)

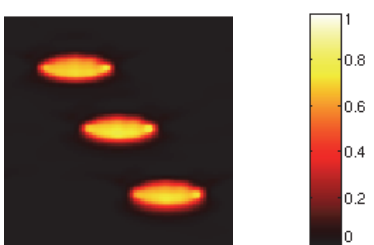

(d)

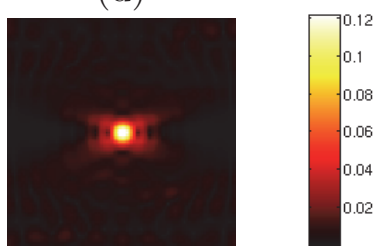

(h)

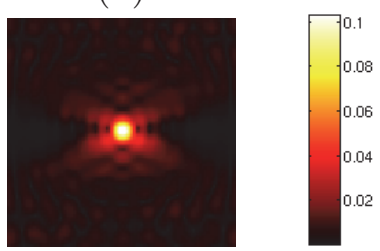

(1)

Figure 4. Blind restoration results on the test image $C$ : a simulated $C L S M$ image. Only $(Y, Z)$ sections of the $3 D$ volumes are presented: (a) original beads, (b) simulated observation, (c) restoration using SGPAM, (d) restoration using RRLAM, (e) first true PSF $h^{1}$, (f) initial PSF ${\hat{h^{1}}}^{(0)}$, (g) estimated $\hat{h}^{1}$ with SGPAM, (h) estimated $\hat{h}^{1}$ with RRLAM, (i) second true PSF $h^{2}$, (j) initial PSF ${\hat{h^{2}}}^{(0)}$, (k) estimated $\hat{h}^{2}$ with $S G P A M$, (l) estimated $\hat{h^{2}}$ with $\operatorname{RRLAM}\left(\alpha=10^{-3}\right.$ and $\left.\beta^{i}=0.1, i=1,2\right)$.

In what follows, we show test results on both the simulated and the real CLSM images. The simulated CLSM image is generated using the theoretical PSF model in [20].

\subsubsection{Test on a simulated CSLM image.}

Image simulation. Consider a 3D image of $100 \times 100 \times 100$ voxels, of three spherical beads, whose axial slice is depicted in Figure 4(a). We denote this image by the letter "C." The beads are assumed to be embedded in a medium of refractive index $n_{s}=1.48$. The CLSM imaging system assumed to have a magnification of $100 X$, a numerical aperture of 1.4 , and an oil immersed lens with a refractive index of $n_{i}=1.5$. The cover-slip is chosen to have a refractive index very close to that of the objective immersion medium $\left(n_{c} \simeq 1.5\right)$ so that aberrations induced by the refractive index mismatch between these two mediums are negligible. The excitation and emission wavelengths are assumed to be $560 \mathrm{~nm}$ and $600 \mathrm{~nm}$, respectively. The pinhole of the confocal microscope is considered to be very small so that it can be approximated by a Dirac function in the PSF generation model of [20]. Radial and axial sampling steps are, respectively, $50 \mathrm{~nm}$ and $145 \mathrm{~nm}$, respecting Nyquist sampling. Knowing all these acquisition parameters, we generate $100 \mathrm{PSF}$, each at a different depth, using the theoretical PSF model in [20]. Then, we generated the blurred image using a discrete version of model (2.1). Adding to it a background noise of $b g=10^{-4}$ and introducing Poisson noise, we obtain the simulated observation whose axial slice is presented in Figure 4(b) with a PSNR 
of $13 \mathrm{~dB}$.

Test and comparison with RRLAM. In order to restore that image, we approximate the depth-variant PSF by a combination of two space invariant PSFs, taken at the top and the bottom of the sample. The considered weighting functions are varying along the $z$-axis and constant along the $x, y$-axis; cf. Figure 1(b), which shows a plot along $z$ of the used weighting functions. The two space invariant PSFs to be estimated are initialized using the theoretical model [20]; parameters included in this model are set in an approximate way (some random errors are introduced in these parameters). The estimated PSFs as well as the recovered image using SGPAM and RRLAM methods are shown in Figures 4(c), (d), (g), (h), (k), and (l), respectively. We also show in Figure 5 (a) the plots of intensity profiles along the $z$-axis passing through the centers of the restored beads and in Figures 5(b) and (c) the intensity profiles along the $z$-axis of the estimated PSFs. Note that the degraded beads are slightly shifted from the original ones due to spherical aberrations; the reconstructed beads are also shifted because this shift is not corrected by the proposed estimation method. One needs further information about the bead shift in order to efficiently estimate it. Numerical values presented in Table 2 show that the result obtained by SGP is more accurate than that obtained by RRLAM. This is because the optimized criterion is not convex, so the algorithms could follow different optimization paths and thus lead to different solutions. The computing time is also lower for the SGPAM method.

Comparison with BD. Comparison with BD given in Table 2 illustrates the advantage of the space variant blur model.

\subsubsection{Test on a real CSLM image.}

Image acquisition. Consider a real image of a plant root of size $200 \times 200 \times 94$ voxels, depicted in Figures 6(a), (e), and (i). We denote this image by the letter "D." This image is observed with a Zeiss LSM 510 microscope having a numerical aperture of 1.3, an oil immersed lens, and a magnification of $40 X$. The radial and axial step-sizes are, respectively, $40 \mathrm{~nm}$ and $140 \mathrm{~nm}$.

Test and comparison with RRLAM. We restored this image using a combination of two space invariant PSFs. The PSFs are initialized using the theoretical PSF model [20] by setting approximately the refractive index of the specimen to 1.33 and of the immersion medium to 1.5. The used weighting functions are varying along the $z$-axis as presented in Figure 1(c). The restored images using the SGPAM and RRLAM methods are displayed, respectively, in Figures 6(b), (f), (j) and (c), (g), (k).

Comparison with BD. We present in Figures $6(\mathrm{~d})$ and $(\mathrm{h})(x, y)$ slices at the top $(z=0)$ and at the bottom $\left(z=z_{\max }\right)$ of the restored image with a space invariant PSF. This result shows that deep slices are not well restored with one space invariant PSF while they are better restored with the proposed approach.

4.3. Discussions. From these experiments, we can say the following.

First, one can notice from Tables 1 and 2 that the SGPAM algorithm provides faster convergence and more accurate results than the RRLAM algorithm. Although one iteration of SGPAM is longer than one iteration of RRLAM, SGPAM converges faster since it requires fewer iterations than RRLAM to reach its convergence. Because of the static step-length value in RRLAM, the algorithm rapidly stagnates to a not necessarily optimal solution. However, 


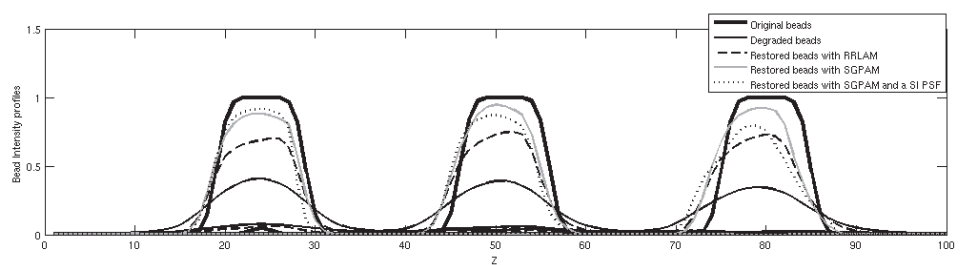

(a)

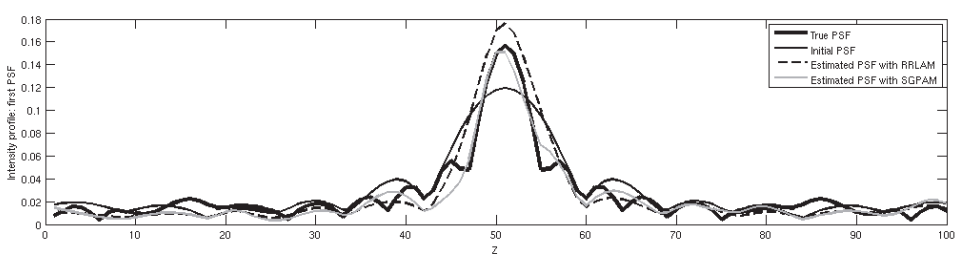

(b)

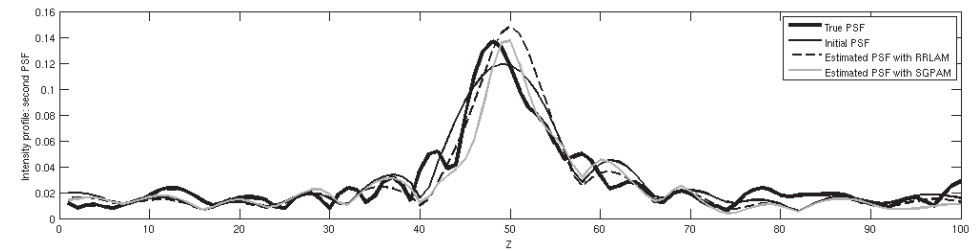

(c)

Figure 5. (a) Intensity profiles along the z-axis passing through the centers of the three beads: the presented plots correspond to the original beads (black-bold line), degraded beads (black-continuous line), restored beads using SGPAM (grey line), restored beads using RRLAM (black-dashed line), and restored beads using a space invariant PSF and SGPAM method (black-dotted line). (b) and (c) Intensity profiles along the z-axis of the first and second space invariant PSFs taken, respectively, at the top and the bottom of the considered sample. True PSFs are presented with the black-bold line, initial PSFs are presented with the black-continuous line, estimated PSFs with SGPAM are presented with the grey line, and estimated PSFs with RRLAM are presented with the black-dashed line.

Table 2

SGPAM method vs. RRLAM method for space variant and space invariant PSF. Tests are presented for $3 D$ images of confocal microscopy. $t_{m}$ is the mean computing time per iteration, and $t_{g}$ is the global computing time.

\begin{tabular}{|l|c|r|c|c|c|c|c|r|}
\hline & \multicolumn{4}{|c|}{ Image C } & \multicolumn{4}{c|}{ Image D } \\
\hline & \multicolumn{2}{|c|}{ RRLAM } & \multicolumn{2}{c|}{ SGPAM } & \multicolumn{2}{c|}{ RRLAM } & \multicolumn{2}{c|}{ SGPAM } \\
\hline & SI & SV & \multicolumn{1}{c|}{ SI } & SV & SI & SV & SI & SV \\
\hline RRE (\%) & 20.17 & 16.59 & 16.03 & 7.81 & - & - & - & - \\
\hline SSIM & 0.85 & 0.88 & 0.90 & 0.95 & - & - & - & - \\
\hline$t_{m}(\min )$ & 0.21 & 0.35 & 0.22 & 0.36 & 0.33 & 0.5 & 0.76 & 1.6 \\
\hline$t_{g}(\min )$ & 6.90 & 9.10 & 6.7 & 6.18 & 5.02 & 14.4 & 5.37 & 11.8 \\
\hline nb it & 32 & 17 & 30 & 26 & 16 & 23 & 7 & 9 \\
\hline
\end{tabular}

in the SGPAM method, thanks to the dynamic step-length selection, SGPAM gives more accurate results than RRLAM. To illustrate this analysis, we present in Figure 7 the global energy variation with the iterations for the test image A.

Second, from the presented tests, one can remark that the proposed blind restoration 


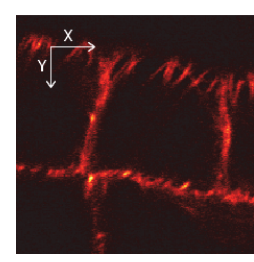

(a)

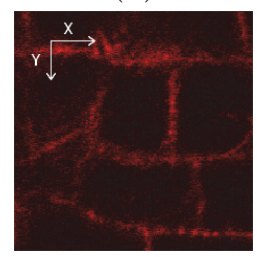

(e)

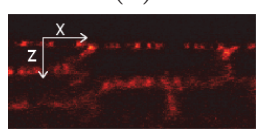

(i)

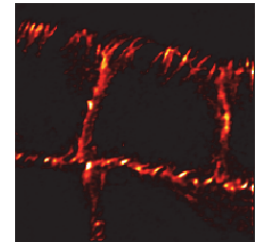

(b)

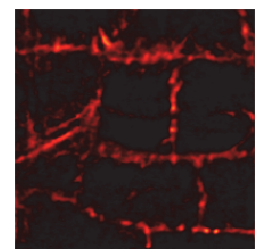

(f)

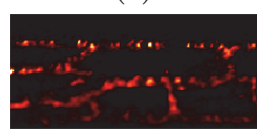

(j)

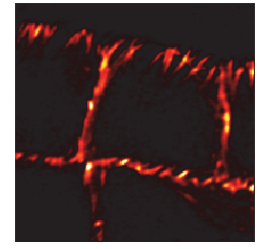

(c)

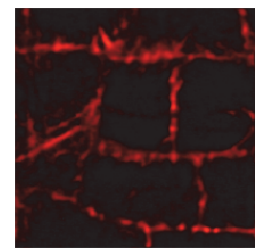

(g)

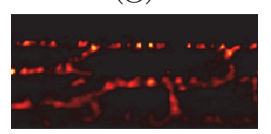

(k)

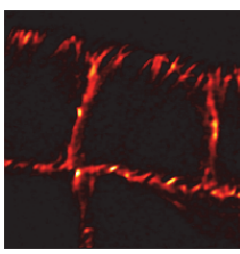

(d)

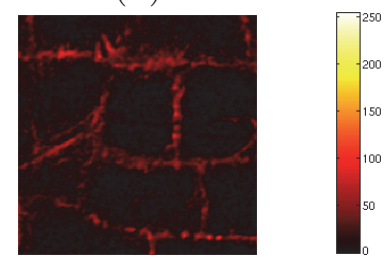

(h)

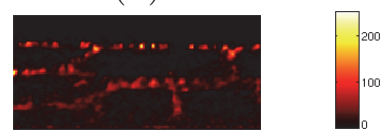

(1)

Figure 6. Blind restoration results on the test image D: a plant root image provided by G. Engler (used with permission) at INRA with a Zeiss LSM 510 microscope. The first and the second raws show, respectively, $(X, Y)$ slices at the top ( $5 \mu \mathrm{m}$ of depth) and the bottom of the sample (10 $\mu \mathrm{m}$ of depth); the third raw shows $(X, Z)$ slices. The first column $((\mathrm{a})$ and $(\mathrm{e}))$ corresponds to the observation, the second column $((\mathrm{b})$ and $(\mathrm{f}))$ corresponds to the restoration using SGPAM, the third column ((c) and $(\mathrm{g}))$ corresponds to the restoration using RRLAM method, and the fourth column $((\mathrm{d})$ and $(\mathrm{h}))$ corresponds to the restoration using a space invariant $P S F$ and SGPAM method $\left(\alpha=0.05\right.$ and $\left.\beta^{i}=10, i=1,2\right)$.

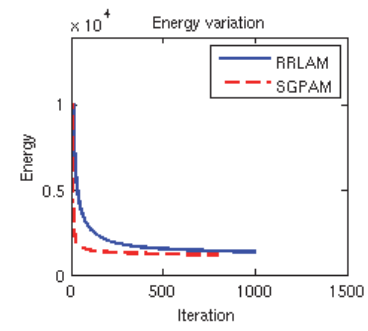

Figure 7. Energy variation with iterations for the test image A.

gives more accurate result than BD when the blur variation is significant. This is illustrated in Figure 5(a), which shows the intensity profiles passing through the centers of each of the three restored beads using a space variant PSF (grey line) and a space invariant PSF (blackdotted line). In particular, the last bead is not well restored by the BD algorithm since the blur at the bottom of the sample (i.e., for high $z$ ) is more significant than at the top of the sample (i.e., for low $z$ ). This also can be seen on the PSF images presented in Figures 4(e) and (i) as well as their intensity profiles along the $z$-axis in Figures $5(\mathrm{~b})$ and (c). One can notice that the second true PSF (Figure 5(c)) is more spread out than the first PSF (Figure $5(\mathrm{~b}))$. Furthermore, using the proposed blind restoration approach, one can efficiently recover the spread of these two different PSFs (see grey plots in Figures 5(b) and (c)). 
5. Conclusion. We proposed a blind restoration method for Poissonian space variant blurred images. The space variant PSF is approximated by a convex combination of a set of space invariant PSFs. The latter is estimated simultaneously with the image by optimizing a regularized criterion including different constraints on the unknown variables (positiveness of the image and the PSFs, PSF normalization, and flux conservation). Numerical tests of the proposed method show encouraging results. The main limitation of the proposed method is that the number of PSFs and weighting functions should be adjusted by the user. Further investigations about the choice of these parameters and their impact on the accuracy of the solution, in a completely blind framework, would be an interesting future work.

Appendix A. Existence of a minimizer for problem (2.16). Our goal is to prove the existence of a solution $\left(\hat{f}, \hat{h}^{1}, \ldots, h^{M}\right) \in \mathcal{F}_{c} \times \mathcal{H}^{M}$ of problem (2.16).

Thanks to the classical method of the calculus of variations [2], the main steps of the proof are

- to show that any minimizing sequence is bounded in $\mathcal{F}_{c} \times \mathcal{H}^{M}$,

- to extract a converging subsequence by using compactness properties, and

- to pass to the limit in the functional by continuity.

So for our problem, it suffices to show the existence of a minimizing sequence $\left(f_{n}, h_{n}^{1}, \ldots\right.$, $\left.h_{n}^{M}\right)_{n}$ of functional $J$ that converges to $\left(f_{*}, h_{*}^{1}, \ldots, h_{*}^{M}\right) \in \mathcal{F}_{c} \times \mathcal{H}^{M}$ such that

$$
\lim _{n \rightarrow+\infty} J\left(f_{n}, h_{n}^{1}, \ldots, h_{n}^{M}\right) \geq J\left(f_{*}, h_{*}^{1}, \ldots, h_{*}^{M}\right) .
$$

For this end, some necessary lemmas should be proved.

Lemma A.1. There exist $a>0$ and $b \in \mathbb{R}$ such that $\forall t>0, \forall j \in \mathcal{I}_{d}$, we have

$$
t-g(j) \log (t) \geq a t+b
$$

Proof. Let us define the following function for fixed $a$ and $b$ in $\mathbb{R}$ :

$$
F(t)=(a-1) t+g(j) \log (t)+b \quad \forall t>0, \forall j \in \mathcal{I}_{d}
$$

The first derivative of this function is given by $F^{\prime}(t)=a-1+\frac{g(j)}{t}$. We know that $F^{\prime}(t) \geq$ $0 \Leftrightarrow \frac{g(j)}{t} \geq 1-a$. Consequently, for $\left.a \in\right] 0,1\left[\right.$, we have $F^{\prime}(t) \geq 0 \Leftrightarrow \frac{g(j)}{1-a} \geq t$. In other words, $F$ is increasing from $F(0)=-\infty$ to $F\left(\frac{g(j)}{1-a}\right)$ in $\left.] 0, \frac{g(j)}{1-a}\right]$ and decreasing from $F\left(\frac{g(j)}{1-a}\right)$ to $F(+\infty)=-\infty$ in $\left[\frac{g(j)}{1-a},+\infty[\right.$. Furthermore, we have

$$
F\left(\frac{g(j)}{1-a}\right)=b-g(j)(1+\log (1-a)-\log (g(j))) .
$$

Hence, for $a \in] 0,1\left[\right.$, we have $F\left(\frac{g(j)}{1-a}\right) \leq 0 \forall j \in \mathcal{I}_{d}$. If we choose $b \leq \operatorname{Inf}_{j \in \mathcal{I}_{d}} g(j)(1+\log (1-a)-$ $\log (g(j)))$, we have $F(t) \leq 0$, i.e., $t-g(j) \log (t) \geq a t+b \forall t>0, \forall j \in \mathcal{I}_{d}$.

By choosing $t=\sum_{1 \leq i \leq M} h^{i} *\left(\psi^{i} . f\right)(u)+b_{g}$ in Lemma A.1, we easily deduce the following 
corollary.

Corollary A.2. From the previous lemma, we can deduce that

$$
\begin{aligned}
J(f, h) & \geq\left(a b_{g}+b\right)\left|\mathcal{I}_{d}\right|+a \sum_{j \in \mathcal{I}_{d}}\left(\sum_{1 \leq i \leq M} \int_{R_{j}} h^{i} *\left(\psi^{i} \cdot f\right)(u) \mathrm{d} u\right) \\
& +\alpha \int_{\mathcal{I}}|D f|+\sum_{1 \leq i \leq M} \beta^{i}\left\|\nabla h^{i}\right\|_{2}^{2},
\end{aligned}
$$

where $\left|\mathcal{I}_{d}\right|$ is the measure of $\mathcal{I}_{d}$.

Now, let $\left(f_{n}, h_{n}^{1}, \ldots, h_{n}^{M}\right) \in \mathcal{F}_{c} \times \mathcal{H}^{M}$ be a minimizing sequence of $J\left(f, h^{1}, \ldots, h^{M}\right)$, i.e., $\left(f_{n}, h_{n}^{1}, \ldots, h_{n}^{M}\right)$ verifies

$$
\lim _{n \rightarrow+\infty} J\left(f_{n}, h_{n}^{1}, \ldots, h_{n}^{M}\right)=\inf _{\mathcal{F}_{c} \times \mathcal{H}^{M}} J\left(f, h^{1}, \ldots, h^{M}\right) .
$$

Moreover, we assume that this infimum is finite. That is, there exists a constant $K_{1}>0$ such that $J\left(f_{n}, h_{n}^{1}, \ldots, h_{n}^{M}\right) \leq K_{1}$. Hence, from this assumption and (A.5), we deduce the following lemma.

Lemma A.3. There exist constants $C_{1}>0$ and $C_{2}>0$ such that

$$
\begin{gathered}
\left\|\nabla h_{n}^{i}\right\|_{2}^{2} \leq C_{1}, 1 \leq i \leq M, \\
\int_{\mathcal{I}}\left|D f_{n}\right| \leq C_{1}, \\
\sum_{j \in \mathcal{I}_{d}} \int_{R_{j}} h_{n}^{i} *\left(\psi^{i} \cdot f_{n}\right)(u) d u \leq C_{2}, 1 \leq i \leq M .
\end{gathered}
$$

Corollary A.4. For $1 \leq i \leq M$, there exist $h_{*}^{i} \in \mathcal{H}$ and a subsequence of $\left(h_{n}^{i}\right)_{n}$ which we also denote by $\left(h_{n}^{i}\right)_{n}$ that weakly converges to $h_{*}^{i}$ in $H^{1}(\mathcal{I})$ and strongly converges to $h_{*}^{i}$ in $L^{2}(\mathcal{I})$.

Proof. We know that each $h_{n}^{i}, 1 \leq i \leq M$, is bounded in $L^{1}(\mathcal{I})$ since $h_{n}^{i} \geq 0$ and $\int_{\mathcal{I}} h_{n}^{i}(u) d u=1$. From the Poincaré inequality, we have $\left|h_{n}^{i}-\frac{1}{|\mathcal{I}|} \int_{\mathcal{I}} h_{n}^{i}(u) d u\right|_{L^{2}(\mathcal{I})} \leq C\left|\nabla h_{n}^{i}\right|_{L^{2}(\mathcal{I})}$ with $C$ a positive constant. From inequality (A.7), we deduce that

$$
\left|h_{n}^{i}-\frac{1}{|\mathcal{I}|} \int_{\mathcal{I}} h_{n}^{i}(u) d u\right|_{L^{2}(\mathcal{I})} \leq C C_{1} .
$$

Hence, $h_{n}^{i}$ is bounded in $L^{2}(\mathcal{I})$. Thus, there exist $h_{*}^{i} \in H^{1}(\mathcal{I})$ and a subsequence of $h_{n}^{i}$ that weakly converges to $h_{*}^{i}$ in $H^{1}(\mathcal{I})$ and strongly converges to $h_{*}^{i}$ in $L^{2}(\mathcal{I})$. Furthermore, we can easily prove that $h_{*}^{i} \in \mathcal{H}$ since we can easily verify by taking the limit that $h_{*}^{i} \geq 0$, $\int_{\mathcal{I}} h_{*}^{i}(u) d u=1$, and $\operatorname{supp}\left(h_{*}^{i}\right) \subset B(O, R)$.

To show that $f_{n}$ is bounded in $L^{1}(\mathcal{I})$ we need the following lemma.

Lemma A.5. Let $h \in \mathcal{H}$, and let $v \in L^{2}(\mathcal{I})$, I-periodic; then we have

$$
|h * v|_{L^{1}(\mathcal{I})} \leq|v|_{L^{1}(\mathcal{I})}
$$


Proof. Let $v \in L^{2}(\mathcal{I}), \mathcal{I}$-periodic; then we have

$$
\begin{aligned}
|h * v|_{L^{1}(\mathcal{I})} & =\int_{\mathcal{I}}|h * v(u)| d u \\
& =\int_{\mathcal{I}}\left|\int_{\mathbb{R}^{2}} h\left(u-u^{\prime}\right) v\left(u^{\prime}\right) d u^{\prime}\right| d u \\
& \leq \int_{\mathcal{I}} \int_{\mathbb{R}^{2}} h\left(u-u^{\prime}\right)\left|v\left(u^{\prime}\right)\right| d u^{\prime} d u .
\end{aligned}
$$

Thanks to the $\mathcal{I}$-periodicity of $\left|v\left(u^{\prime}\right)\right|$ and Lemma 2.1, we get $|h * v|_{L^{1}(\mathcal{I})} \leq \int_{\mathcal{I}}|v(u)| d u$.

Lemma A.6. There exists a constant $C_{4}$ such that $\left|f_{n}\right|_{L^{1}(\mathcal{I})} \leq C_{4}$.

Proof. Now, we focus on proving that the sequence $\left(f_{n}\right)_{n}$ is bounded. For that, let us consider the following two functions:

$$
\begin{aligned}
w_{n}= & \frac{1}{\mathcal{I}} \int_{\mathcal{I}} f_{n}(u) d u, \\
& v_{n}=f_{n}-w_{n} .
\end{aligned}
$$

We can easily verify that $\int_{\mathcal{I}} v_{n}(u) d u=0$ and $D v_{n}=D f_{n}$. Consequently, from (A.8), we obtain $\int_{\mathcal{I}}\left|D v_{n}(u)\right|=\int_{\mathcal{I}}\left|D f_{n}\right| \leq C_{1}$. Furthermore, from the Poincaré inequality, there exists a constant $C>0$ such that

$$
\left|v_{n}-\frac{1}{|\mathcal{I}|} \int_{\mathcal{I}} v_{n}(u) d u\right|_{L^{1}(\mathcal{I})} \leq C\left|D v_{n}\right|_{L^{1}(\mathcal{I})}
$$

Hence, since $\int_{\mathcal{I}} v_{n}(u) d u=0$, we have

$$
\left|v_{n}\right|_{L^{1}(\mathcal{I})} \leq C_{3} \text { with } C_{3}=C C_{1} .
$$

Besides, we know that $h_{n}^{i} * \psi^{i} w_{n}=h_{n}^{i} * \psi^{i} f_{n}-h_{n}^{i} * \psi^{i} v_{n}$. Hence, we obtain

$$
\left|h_{n}^{i} * \psi^{i} w_{n}\right|_{L^{1}(\mathcal{I})} \leq\left|h_{n}^{i} * \psi^{i} f_{n}\right|_{L^{1}(\mathcal{I})}+\left|h_{n}^{i} * \psi^{i} v_{n}\right|_{L^{1}(\mathcal{I})} .
$$

Thanks to PSF normalization and Lemma 2.1, we have $\left|h_{n}^{i} * \psi^{i} f_{n}\right|_{L^{1}(\mathcal{I})}=\left|\psi^{i} f_{n}\right|_{L^{1}(\mathcal{I})} \leq$ $\left|f_{n}\right|_{L^{1}(\mathcal{I})}=c$. Hence, $\left|h_{n}^{i} * \psi^{i} w_{n}\right|_{L^{1}(\mathcal{I})} \leq c+\left|v_{n}\right|_{L^{1}(\mathcal{I})}$. From (A.16), we get $\forall i \in\{1, \ldots, M\}$,

$$
\left|h_{n}^{i} * \psi^{i} w_{n}\right|_{L^{1}(\mathcal{I})} \leq c+C_{3}=C_{4} .
$$

Besides, thanks to the periodicity of $\psi^{i}$, we can prove that

$$
\left|h_{n}^{i} * \psi^{i} w_{n}\right|_{L^{1}(\mathcal{I})}=\int_{\mathcal{I}} f_{n}(u) d u
$$

Indeed,

$$
\left|h_{n}^{i} * \psi^{i} w_{n}\right|_{L^{1}(\mathcal{I})}=\int_{\mathcal{I}}\left|\int_{\mathbb{R}^{n}} h_{n}\left(u-u^{\prime}\right) \psi^{i} w_{n}\left(u^{\prime}\right) d u^{\prime}\right| d u .
$$


Since $h_{n}^{i}, \psi^{i}$, and $w_{n}$ are positive, we obtain

$$
\begin{aligned}
\left|h_{n}^{i} * \psi^{i} w_{n}\right|_{L^{1}(\mathcal{I})} & =\int_{\mathcal{I}} \int_{\mathbb{R}^{n}} h_{n}\left(u-u^{\prime}\right) \psi^{i}\left(u^{\prime}\right) w_{n}\left(u^{\prime}\right) d u^{\prime} d u \\
& =\frac{1}{|\mathcal{I}|} \int_{\mathcal{I}} f_{n}\left(u^{\prime}\right) d u^{\prime} \int_{\mathcal{I}} \int_{\mathbb{R}^{n}} h_{n}\left(u-u^{\prime}\right) \psi^{i}\left(u^{\prime}\right) d u^{\prime} d u .
\end{aligned}
$$

Since $\int_{\mathbb{R}^{2}} h_{n}^{i}\left(u-u^{\prime}\right) d u^{\prime}=1 \forall u \in \mathcal{I}$ and $\psi^{i}$ is $\mathcal{I}$-periodic, we get using Lemma 2.1 the following result:

$$
\left|h_{n}^{i} * \psi^{i} w_{n}\right|_{L^{1}(\mathcal{I})}=\frac{1}{|\mathcal{I}|} \int_{\mathcal{I}} f_{n}(u) d u \int_{\mathcal{I}} \psi^{i}(u) d u
$$

Summing over $i$ and taking into account that $\sum_{1 \leq i \leq M} \psi^{i}(u)=1 \forall u \in \mathcal{I}$, we have

$$
\begin{aligned}
\sum_{1 \leq i \leq M}\left|h_{n}^{i} * \psi^{i} w_{n}\right|_{L^{1}(\mathcal{I})} & =\frac{1}{|\mathcal{I}|} \int_{\mathcal{I}} f_{n}(u) d u \int_{\mathcal{I}} \sum_{1 \leq i \leq M} \psi^{i}(u) d u \\
& =\int_{\mathcal{I}} f_{n}(u) d u
\end{aligned}
$$

Consequently, $\int_{\mathcal{I}} f_{n}(u) d u \leq C_{4}$, i.e., $\left|f_{n}\right|_{L^{1}(\mathcal{I})} \leq C_{4}$.

We proved that $f_{n}$ is bounded in $B V(\mathcal{I})$, i.e., $\left|f_{n}\right|_{L^{1}} \leq C_{4}$ and $\left|D f_{n}\right|_{L^{1}} \leq C_{1}$. Consequently, there exists $f_{*}$ such that $f_{n}$ weakly converges to $f_{*}$ in $B V(\mathcal{I})$ and strongly converges to $f_{*}$ in $L^{1}(\mathcal{I})$ since $B V(\mathcal{I})$ is compactly embedded in $L^{1}(\mathcal{I})$; see [2].

Now, let us prove that $f_{*} \in \mathcal{F}_{c}$. By taking the limit of $f_{n}$, it is easy to show that $\int_{\mathcal{I}} f_{*}(u) d u=c$. It suffices to show that $f_{*}$ is $\mathcal{I}$-periodic. For the sake of clarity, we present the proof in the $1 \mathrm{D}$ case, i.e., $\mathcal{I}=[-1,1]$; the considered period is thus 2 .

Let $\widetilde{f}_{*}$ be the $\mathcal{I}$-periodic extension of $f_{*}$, and let $\phi \in L^{1}(\mathcal{I})$. We show that for each cell $\mathcal{I}_{k}=[-1+2 k, 1+2 k], k \in \mathbb{Z}, f_{n}$ weakly converges to $\widetilde{f}_{*}$ in $L^{1}(\mathcal{I})$ :

$$
\begin{aligned}
\lim _{n \rightarrow+\infty} \int_{-1+2 k}^{1+2 k} f_{n}(u) \phi(u) d u & =\lim _{n \rightarrow+\infty} \int_{-1}^{1} f_{n}(u+2 k) \phi(u+2 k) d u \\
& =\lim _{n \rightarrow+\infty} \int_{-1}^{1} f_{n}(u) \phi(u+2 k) d u \\
& =\int_{-1}^{1} f_{*}(u) \phi(u+2 k) d u \\
& =\int_{-1+2 k}^{1+2 k} \widetilde{f}_{*}(u+2 k) \phi(u) d u \\
& =\int_{-1+2 k}^{1+2 k} \widetilde{f}_{*}(u) \phi(u) d u .
\end{aligned}
$$

Thus, $f_{n}$ weakly converges to $\widetilde{f}_{*}$ in $L^{1}(\mathcal{I})$. In a similar way, we prove the periodicity of $D f_{*}$.

Theorem A.7. The problem $\operatorname{Inf}_{\mathcal{F}_{c} \times \mathcal{H}^{M}} J\left(f, h^{1}, \ldots, h^{M}\right)$ has at least one solution. 
Proof. Thanks to Lemma A.6, we have that any minimizing sequence $\left(f_{n}, h_{n}^{1}, \ldots, h_{n}^{M}\right)_{n}$ converges (up to a subsequence) to $\left(f_{*}, h_{*}^{1}, \ldots, h_{*}^{M}\right) \in \mathcal{F}_{c} \times \mathcal{H}^{M}$. We have to prove that $\liminf _{n \rightarrow+\infty} J\left(f_{n}, h_{n}^{1}, \ldots, h_{n}^{M}\right) \geq J\left(f_{*}, h_{*}^{1}, \ldots, h_{*}^{M}\right)$.

From the convexity of functions $\int_{\mathcal{I}}\left|\nabla h^{i}(u)\right|^{2} d u, 1 \leq i \leq M$, and $\int_{\mathcal{I}}|D f(u)|$, we have

$$
\begin{array}{r}
\liminf _{n \rightarrow+\infty} \int_{\mathcal{I}}\left|\nabla h_{n}(u)\right|^{2} d u \geq \int_{\mathcal{I}}\left|\nabla h_{*}(u)\right|^{2} d u, \\
\liminf _{n \rightarrow+\infty} \int_{\mathcal{I}}\left|D f_{n}\right| \geq \int_{\mathcal{I}}\left|D f_{*}\right| d u .
\end{array}
$$

It remains to show that

$$
\begin{array}{r}
\liminf _{n \rightarrow+\infty} \sum_{j \in \mathcal{I}_{d}} G\left(\sum_{1 \leq i \leq M} \int_{R_{j}} h_{n}^{i} *\left(\psi^{i} \cdot f_{n}\right)(u) \mathrm{d} u+b_{g}\right) \\
\geq \sum_{j \in \mathcal{I}_{d}} G\left(\sum_{1 \leq i \leq M} \int_{R_{j}} h_{*}^{i} *\left(\psi^{i} \cdot f_{*}\right)(u) \mathrm{d} u+b_{g}\right),
\end{array}
$$

with $G(t)=t-\mathrm{g}(j) \log (t) \forall t>0$ and $j \in \mathcal{I}_{d}$.

From the strong convergence of $h_{n}^{i} *\left(\psi^{i} . f_{n}\right)$ to $h_{*}^{i} *\left(\psi^{i} . f_{*}\right)$ in $L^{1}(\mathcal{I})$, we deduce that

$$
\lim _{n \rightarrow+\infty} \int_{R_{j}} h_{n}^{i} *\left(\psi^{i} \cdot f_{n}\right)(u) \mathrm{d} u+b_{g}=\int_{R_{j}} h_{*}^{i} *\left(\psi^{i} \cdot f_{*}\right)(u) \mathrm{d} u+b_{g}
$$

From the continuity of $G$, we have

$$
\begin{array}{r}
\lim _{n \rightarrow+\infty} \sum_{j \in \mathcal{I}_{d}} G\left(\sum_{1 \leq i \leq M} \int_{R_{j}} h_{n}^{i} *\left(\psi^{i} \cdot f_{n}\right)(u) \mathrm{d} u+b_{g}\right) \\
=\sum_{j \in \mathcal{I}_{d}} G\left(\sum_{1 \leq i \leq M} \int_{R_{j}} h_{*}^{i} *\left(\psi^{i} \cdot f_{*}\right)(u) \mathrm{d} u+b_{g}\right) .
\end{array}
$$

Thereby, we proved that $\liminf _{n \rightarrow+\infty} J\left(f_{n}, h_{n}^{1}, \ldots, h_{n}^{M}\right) \geq J\left(f_{*}, h_{*}^{1}, \ldots, h_{*}^{M}\right)$ and hence we showed that $\left(f_{*}, h_{*}^{1}, \ldots, h_{*}^{M}\right) \in \arg \min _{\mathcal{F}_{c} \times \mathcal{H}^{M}} J\left(f, h^{1}, \ldots, h^{M}\right)$.

Appendix B. Gradient decomposition. We give here a decomposition of the gradient of the used regularizing terms for both $2 \mathrm{D}$ and $3 \mathrm{D}$ images. In this paper, the considered regularizing terms are the following:

- for $2 \mathrm{D}$ images $X \in \mathbb{R}^{n} \times \mathbb{R}^{n}$ :

$$
J^{R}(X)=\sum_{1 \leq i, j \leq n} \phi\left(D_{i, j}^{2}\right)
$$

where $D_{i, j}^{2}=\frac{1}{2}\left|(\nabla X)_{i, j}\right|^{2}$ and the discrete gradient $(\nabla X)_{i, j}$ is computed by adding one column and one row to $X$ as follows:

$$
(\nabla X)_{i, j}=\left(\begin{array}{c}
X_{i+1, j}-X_{i, j} \\
X_{i, j+1}-X_{i, j}
\end{array}\right)
$$

with $X_{n+1, j}=X_{1, j}$ and $X_{i, n+1}=X_{i, 1}$; 
- for 3D images $X \in \mathbb{R}^{n} \times \mathbb{R}^{n} \times \mathbb{R}^{n}$ :

$$
J^{R}(X)=\sum_{1 \leq i, j \leq n} \phi\left(D_{i, j, k}^{2}\right)
$$

where $D_{i, j, k}^{2}=\frac{1}{2}\left|(\nabla X)_{i, j, k}\right|^{2}$ and the discrete gradient $(\nabla X)_{i, j, k}$ is as follows:

$$
(\nabla X)_{i, j, k}=\left(\begin{array}{c}
X_{i+1, j, k}-X_{i, j, k} \\
X_{i, j+1, k}-X_{i, j, k} \\
X_{i, j, k+1}-X_{i, j, k}
\end{array}\right)
$$

with $X_{n+1, j, k}=X_{1, j, k}, X_{i, n+1, k}=X_{i, 1, k}$, and $X_{i, j, n+1}=X_{i, j, n+1}$

The function $\phi(t)$ is chosen as $\phi(t)=2 \sqrt{t+\epsilon^{2}} \forall t \geq 0, \epsilon>0$ for the total variation term used in (3.11) and as $\phi(t)=t \forall t \geq 0$ for the quadratic regularizing term used in (3.20). The parameter $\epsilon$ is a very small scalar used to avoid singularity points in the derivative of the total variation term (e.g., in our simulation we consider $\epsilon=10^{-8}$ as suggested in [41, 47]).

Now, let us first consider 2D images. The gradient of function (B.1) w.r.t. $X_{i, j}$ is expressed as

$$
\begin{aligned}
\nabla_{i, j} J^{R}(X) & =\phi^{\prime}\left(D_{i, j}^{2}\right)\left(2 X_{i, j}-X_{i+1, j}-X_{i, j+1}\right)+\phi^{\prime}\left(D_{i-1, j}^{2}\right)\left(X_{i, j}-X_{i-1, j}\right) \\
& +\phi^{\prime}\left(D_{i, j-1}^{2}\right)\left(X_{i, j}-X_{i, j-1}\right),
\end{aligned}
$$

with $\phi^{\prime}(t)$ the derivative of $\phi(t)$. A possible decomposition of the gradient $\nabla_{i, j} J^{R}(x)$ into two positive terms is as follows:

$$
\begin{gathered}
U^{R}(X)=\phi^{\prime}\left(D_{i, j}^{2}\right)\left(X_{i+1, j}+X_{i, j+1}\right)+\phi^{\prime}\left(D_{i-1, j}^{2}\right) X_{i-1, j}+\phi^{\prime}\left(D_{i, j-1}^{2}\right) X_{i, j-1}, \\
V^{R}(X)=\left[2 \phi^{\prime}\left(D_{i, j}^{2}\right)+\phi^{\prime}\left(D_{i-1, j}^{2}\right)+\phi^{\prime}\left(D_{i, j-1}^{2}\right)\right] X_{i, j} .
\end{gathered}
$$

Now, we give similar expressions for 3D images:

$$
\begin{aligned}
U^{R}(X) & =\phi^{\prime}\left(D_{i, j, k}^{2}\right)\left(X_{i+1, j, k}+X_{i, j+1, k}+X_{i, j, k+1}\right)+\phi^{\prime}\left(D_{i-1, j, k}^{2}\right) X_{i-1, j, k} \\
& +\phi^{\prime}\left(D_{i, j-1, k}^{2}\right) X_{i, j-1, k}+\phi^{\prime}\left(D_{i, j, k-1}^{2}\right) X_{i, j, k-1}, \\
V^{R}(X) & =X_{i, j, k}\left[2 \phi^{\prime}\left(D_{i, j, k}^{2}\right)+\phi^{\prime}\left(D_{i-1, j, k}^{2}\right)+\phi^{\prime}\left(D_{i, j-1, k}^{2}\right)+\phi^{\prime}\left(D_{i, j, k-1}^{2}\right)\right] .
\end{aligned}
$$

Acknowledgments. We acknowledge Praveen Pankajakshan and Gilbert Engler for collaborating with us within the ANR Diamond project. We also acknowledge ATE for important interactions within the FUI Gyrovision project and for providing images for digital camera tests. Finally, we acknowledge Roberto Cavicchioli for providing us SGP code and Praveen Pankajakshan for confocal PSF code. 


\section{REFERENCES}

[1] M. Arigovindan, J. Shaevitz, J. McGowan, J.W. Sedat, and D.A. Agard, A parallel productconvolution approach for representing the depth varying point spread functions in $3 d$ widefield microscopy based on principal component analysis, Optics Express, 18 (2010), pp. 6461-6476.

[2] G. Aubert and P. Kornprobst, Mathematical Problems in Image Processing: Partial Differential Equations and the Calculus of Variations, Appl. Math. Sci. 147, Springer-Verlag, New York, 2006.

[3] L. BAR, N. Sochen, AND N. KIRYATI, Restoration of images with piecewise space-variant blur, in Scale Space and Variational Methods in Computer Vision, Springer-Verlag, Berlin, 2007, pp. 533-544.

[4] J. Bardsley, S. Jefferies, J. Nagy, and R. Plemmons, Blind iterative restoration of images with spatially-varying blur, Optics Express, 14 (2006), pp. 1767-1782.

[5] J. Barzilai And J.M. Borwein, Two-point step size gradient methods, IMA J. Numer. Anal., 8 (1988), pp. 141-148.

[6] S. BeCKer AND J. FAdiLI, A quasi-Newton proximal splitting method, in Advances in Neural Information Processing Systems (NIPS), MIT Press, Cambridge, MA, 2012, pp. 2627-2635.

[7] S. Ben Hadj And L. Blanc-FÉRAud, Modeling and removing depth-variant blur from 3D fluorescence microscopy, in Proceedings of the IEEE International Conference on Acoustics, Speech, and Signal Processing (ICASSP), Kyoto, Japan, 2012.

[8] S. Ben Hadj, L. Blanc-Féraud, G. Aubert, G. Engler, E. Maalouf, B. Colicchio, and A. DiETERLEN, Blind depth-variant blur removal in confocal microscopy, in Proceedings of the IEEE International Symposium on Biomedical Imaging (ISBI), San Francisco, CA, 2013.

[9] M. Blume, D. Zikic, W. Wein, And N. Navab, A new and general method for blind shift-variant deconvolution of biomedical images, in Medical Image Computing and Computer-Assisted Intervention, Lecture Notes in Comput. Sci. 4791, Springer-Verlag, Berlin, 2007, pp. 743-750.

[10] S. BonetTinI, Inexact block coordinate descent methods with application to non-negative matrix factorization, IMA J. Numer. Anal., 31 (2011), pp. 1431-1452.

[11] S. Bonettini, R. Zanella, And L. Zanni, A scaled gradient projection method for constrained image deblurring, Inverse Problems, 25 (2009), 015002.

[12] M. Born and E. Wolf, Principles of Optics, Pergamon Press, Oxford, UK, 1980.

[13] M. Carlavan and L. Blanc-FÉraud, Sparse Poisson noisy image deblurring, IEEE Trans. Image Process., 21 (2012), pp. 1834-1846.

[14] C. Chaux, J.-C. Pesquet, And N. Pustelnik, Nested iterative algorithms for convex constrained image recovery problems, SIAM J. Imaging Sci., 2 (2009), pp. 730-762.

[15] L. Denis, E. Thí́BAut, And F. Soulez, Fast model of space-variant blurring and its application to deconvolution in astronomy, in Proceedings of the IEEE International Conference on Image Processing (ICIP), Brussels, Belgium, 2011.

[16] N. Dey, L. Blanc-Fraud, C. Zimmer, Z. Kam, P. Roux, J.C. Olivo-Marin, and J. Zerubia, Richardson-Lucy algorithm with total variation regularization for $3 d$ confocal microscope deconvolution, Microsc. Res. Tech., 69 (2006), pp. 260-266.

[17] F.X. Dupé, J.M. Fadili, And J.L. Starck, A proximal iteration for deconvolving Poisson noisy images using sparse representations, IEEE Trans. Image Process., 18 (2009), pp. 310-321.

[18] M.A.T. Figueiredo And J.M. Bioucas-Dias, Restoration of Poissonian images using alternating direction optimization, IEEE Trans. Image Process., 19 (2010), pp. 3133-3145.

[19] G. Frassoldati, L. Zanni, And G. Zanghirati, New adaptive stepsize selections in gradient methods, J. Ind. Manag. Optim., 4 (2008), pp. 299-312.

[20] S.F. Gibson And F. LANni, Experimental test of an analytical model of aberration in an oil-immersion objective lens used in three-dimensional light microscopy, J. Opt. Soc. Amer. A, 8 (1991), pp. 16011613.

[21] S.F. Gibson AND F. LANNI, Diffraction by a circular aperture as a model for three-dimensional optical microscopy, J. Opt. Soc. Amer. A, 6 (1989), pp. 1357-1367.

[22] Y.P. Guo, H.P. LeE, AND C.L. TeO, Blind restoration of images degraded by space-variant blurs using iterative algorithms for both blur identification and image restoration, Image Vis. Comput., 15 (1996), pp. 399-410.

[23] Z.T. Harmany, R.F. Marcia, and R.M. Willett, This is SPIRAL-TAP: Sparse Poisson intensity 
reconstruction algorithms - theory and practice, IEEE Trans. Image Process., 21 (2012), pp. 10841096.

[24] S. Harmeling, M. Hirsch, And B. Schlkopf, Space-variant single-image blind deconvolution for removing camera shake, in Advances in Neural Information Processing Systems (NIPS), MIT Press, Cambridge, MA, 2010, pp. 829-837.

[25] M. Hirsch, S. Sra, B. Scholkopf, And S. Harmeling, Efficient filter flow for space-variant multiframe blind deconvolution, in Proceedings of the IEEE Conference on Computer Vision and Pattern Recognition (CVPR), 2010, pp. 607-614.

[26] S. InouÉ, Foundations of confocal scanned imaging in light microscopy, in Handbook of Biological Confocal Microscopy, Springer, New York, 2006, pp. 1-19.

[27] H.W. Kuhn And A.W. Tucker, Nonlinear programming, in Second Berkeley Symposium on Mathematical Statistics and Probability, University of California Press, Berkeley, Los Angeles, 1951, pp. 481-492.

[28] R.L. LagendiJK And J. Biemond, Block-adaptive image identification and restoration, in Proceedings of the IEEE International Conference on Acoustics, Speech, and Signal Processing (ICASSP), 1991, pp. 2497-2500.

[29] J.G. NAgY And D.P. O'LeARY, Restoring images degraded by spatially variant blur, SIAM J. Sci. Comput., 19 (1998), pp. 1063-1082.

[30] P. Pankajakshan, Blind Deconvolution for Confocal Laser Scanning Microscopy, Ph.D. thesis, University of Nice Sophia Antipolis, Nice, France, 2009.

[31] J.B. PAWLEY, Fundamental limits in confocal microscopy, in Handbook of Biological Confocal Microscopy, Springer, New York, 2006, pp. 20-42.

[32] C. Preza And J.A. Conchello, Depth-variant maximum-likelihood restoration for three-dimensional fluorescence microscopy, J. Opt. Soc. Amer., 21 (2004), pp. 1593-1601.

[33] A.N. RaJAgopalan And S. Chaudhuri, MRF model-based identification of shift-variant point spread function for a class of imaging systems, Signal Process., 76 (1999), pp. 285-299.

[34] L.I. Rudin, S. Osher, AND E. FATEMI, Nonlinear total variation based noise removal algorithms, Phys. D, 60 (1992), pp. 259-268.

[35] C.J. Schuler, M. Hirsch, S. Harmeling, And B. Schlkopf, Blind correction of optical aberrations, in Proceedings of the 12th European Conference on Computer Vision, Lecture Notes in Comput. Sci. 7574, Springer-Verlag, Berlin, 2012, pp. 187-200.

[36] S. Setzer, G. Steidl, And T. Teuber, Deblurring Poissonian images by split Bregman techniques, J. Vis. Comm. Image Represent., 21 (2010), pp. 193-199.

[37] L.A. ShePP AND Y. VARDI, Maximum likelihood reconstruction for emission tomography, IEEE Trans. Med. Imag., 1 (1982), pp. 113-122.

[38] J.G. Sivak And R.O. Kreuzer, Spherical aberration of the crystalline lens, Vis. Res., 23 (1983), pp. 5970.

[39] A. Stagliano, P. Boccacci, and M. Bertero, Analysis of an approximate model for Poisson data reconstruction and a related discrepancy principle, Inverse Problems, 27 (2011), 125003.

[40] J.-L. Starck and F. Murtagh, Astronomical Image and Data Analysis, Springer-Verlag, Berlin, Heidelberg, 2006.

[41] C.R. Vogel, Computational Methods for Inverse Problems, Frontiers Appl. Math. 23, SIAM, Philadelphia, 2002.

[42] Z. Wang, A.C. Bovik, H.R. Sheikh, And E.P. Simoncelli, Image quality assessment: From error visibility to structural similarity, IEEE Trans. Image Process., 13 (2004), pp. 600-612.

[43] M. Welk, P. Raudaschl, T. Schwarzbauer, M. Erler, and M. Luter, Fast and robust linear motion deblurring, Signal Image Video P., DOI 10.1007/s11760-013-0563-x, 2013.

[44] Y. XU AND W. YIN, A block coordinate descent method for regularized multiconvex optimization with applications to nonnegative tensor factorization and completion, SIAM J. Imaging Sci., 6 (2013), pp. 1758-1789.

[45] Y.L. You And M. Kaveh, A regularization approach to joint blur identification and image restoration, IEEE Trans. Image Process., 5 (1996), pp. 416-428.

[46] S. YuAN AND C. PReZA, 3D fluorescence microscopy imaging accounting for depth-varying point-spread functions predicted by a strata interpolation method and a principal component analysis method, in International Society for Optics and Photonics (SPIE), Vol. 19, 2011, pp. 23298-23314. 
[47] R. Zanella, P. Boccacci, L. Zanni, and M. Bertero, Efficient gradient projection methods for edge-preserving removal of Poisson noise, Inverse Problems, 25 (2009), 045010.

[48] H. ZHANG AND D. WIPF, Non-uniform camera shake removal using a spatially-adaptive sparse penalty, in Advances in Neural Information Processing Systems (NIPS), MIT Press, Cambridge, MA, 2013, pp. 1556-1564.

[49] X. Zhu And P. Milanfar, Automatic parameter selection for denoising algorithms using a no-reference measure of image content, IEEE Trans. Image Process., 19 (2010), pp. 3116-3132. 